\title{
Summary of the 2008 NASA Fundamental Aeronautics Program Sonic Boom Prediction Workshop
}

\author{
Michael A. Park* Michael J. Aftosmis ${ }^{\dagger}$ \\ Melissa B. Carter $§ \quad$ Susan E. Cliff $₫ \quad$ Linda S. Bangert ॥ \\ Richard L. Campbell ${ }^{\ddagger}$
}

\begin{abstract}
The Supersonics Project of the NASA Fundamental Aeronautics Program organized an internal sonic boom workshop to evaluate near- and mid-field sonic boom prediction capability at the Fundamental Aeronautics Annual Meeting in Atlanta, Georgia on October 8, 2008. Workshop participants computed sonic boom signatures for three non-lifting bodies and two lifting configurations. A cone-cylinder, parabolic, and quartic bodies of revolution comprised the non-lifting cases. The lifting configurations were a simple 69-degree delta wing body and a complete low-boom transport configuration designed during the High Speed Research Project in the 1990s with wing, body, tail, nacelle, and boundary layer diverter components. The AIRPLANE, Cart3D, FUN3D, and USM3D flow solvers were employed with the ANET signature propagation tool, output-based adaptation, and $a$ priori adaptation based on freestream Mach number and angle of attack. Results were presented orally at the workshop. This article documents the workshop, results, and provides context on previously available and recently developed methods.
\end{abstract}

\section{Introduction}

Supersonic flights over land by civil aircraft are currently prohibited in many countries, including the United States of America. The acceptance of an aircraft's sonic boom to the general population and regulatory agencies is a requirement for supersonic flights over land and therefore the commercial viability of a supersonic transport. Predicting how sonic boom signatures are perceived is a challenging task that requires the prediction of the signature on the ground. This is a task complicated by long propagation distances, atmospheric variations, and the Earth's turbulent boundary layer. ${ }^{1}$ A detailed review of the history and state-of-the-art of sonic boom modeling is provided by Plotkin. ${ }^{2}$ Surveys of sonic boom prediction methods are available from Ozcer $^{3}$ and Park. ${ }^{4}$

The propagation of a sonic boom is often separated into two logical stages or regions, depicted in Fig. 1, to facilitate analysis. The near field is a region near the aircraft, where shocks are formed and strongly influenced by nonlinear phenomena such as shock-shock interaction, shock curvature, and cross flow. Computational Fluid Dynamics (CFD) methods are typically employed in this near field region, denoted CFD Domain in Fig. 1, to capture these phenomena. Higher pressure portions of the signature travel faster than lower pressure portions of the signature because of variations in the local speed of sound. This slight speed difference causes the expansions to elongate and the shocks to coalesce as they propagate. Sonic boom propagation methods ${ }^{6}$ are typically employed in the far field where the geometric details of the configuration are less important

\footnotetext{
*Research Scientist, Computational AeroSciences Branch, NASA Langley Research Center, MS 128, Hampton, VA 23681, AIAA Senior Member.

${ }^{\dagger}$ Aerospace Engineer, Modeling and Simulation Branch, NASA Ames Research Center, MS 258-5, Moffett Field, CA 94035, AIAA Associate Fellow.

${ }^{\ddagger}$ Senior Research Engineer, Configuration Aerodynamics Branch, NASA Langley Research Center, MS 499, Hampton, VA 23681, AIAA Associate Fellow.

$\S$ Aerospace Engineer, Configuration Aerodynamics Branch, NASA Langley Research Center, MS 499, Hampton, VA 23681, AIAA Senior Member.

ฯAerospace Engineer, Applied Modeling and Simulation Branch, NASA Ames Research Center, MS 258-2, Moffett Field, CA 94035, AIAA Associate Fellow.

"| Aerospace Engineer, Configuration Aerodynamics Branch, NASA Langley Research Center, MS 499, Hampton, VA 23681, AIAA Associate Fellow.
} 


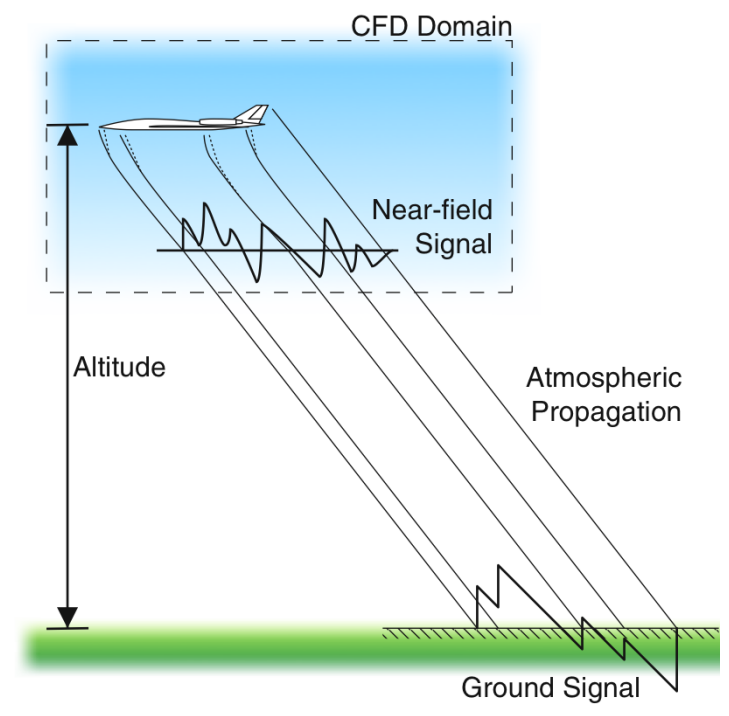

Figure 1. Sonic boom signal propagation. ${ }^{5}$

than atmospheric variations and molecular relaxation phenomena. These atmospheric propagation methods rely on the availability of an accurate near-field signature.

The NASA Fundamental Aeronautics Program Supersonics Project held a workshop at the 2008 Fundamental Aeronautics Annual Meeting in Atlanta, Georgia to evaluate NASA's capability to predict the near-field and mid-field signatures of simple and complex geometries. The participants provided method descriptions at the workshop and a summary presentation was prepared. Table 1 is the workshop agenda listing the duration, titles, and authors of the oral presentations. Table 2 is a listing of the models considered in the workshop. This article summarizes the results presented at the 2008 workshop as well as places the workshop in the context of previous work and developments that took place after the workshop.

Table 1. 2008 NASA Fundamental Aeronautics Program Sonic Boom Prediction Workshop agenda.

15 min. Introduction and Case Descriptions for the Sonic Boom Prediction Workshop Susan Cliff, NASA Ames

45 min. Assessment of Unstructured Euler Methods for Sonic Boom Pressure Signatures Using Grid Refinement and Domain Rotation Methods

Susan Cliff, Scott Thomas, Matt McMullen, John Melton, and Don Durston, NASA Ames

30 min. Output-Adaptive Tetrahedral Cut-Cell Validation for Sonic Boom Prediction

Michael Park and Eric Nielsen, NASA Langley

30 min. Sonic-Boom Prediction with Output-Based Adaptation and Cart3D

Michael Aftosmis, Marian Nemec, and Mathias Wintzer, NASA Ames

30 min. A Method for Shearing and Stretching Unstructured Grids for Improved Sonic Boom Prediction

Richard Campbell and Melissa Carter, NASA Langley

30 min. Summary and Comparison of NASA's Supersonic Boom Prediction Methods Melissa Carter, NASA Langley 
Table 2. Sonic Boom Prediction Workshop models.

\begin{tabular}{lllll} 
Configuration & Mach & $\begin{array}{c}\text { Angle of } \\
\text { Attack }\end{array}$ & h/l & Report \\
\hline 6.48 degree Cone-Cylinder & 1.68 & 0.0 & 10.0 & NASA TM X-22197 \\
Parabolic Body of Revolution & 1.41 & 0.0 & 10.0 & NASA TN D-3106 \\
Quartic Body of Revolution & 1.41 & 0.0 & 10.0 & NASA TN D-3106 \\
69-degree Swept Delta-Wing-Body & 1.68 & 4.74 & 3.6 & NASA TN D-7160 \\
Ames Low Boom Wing-Body-Tail & 2.0 & 2.0 & 1.167 & NASA CP-1999-209699
\end{tabular}

(LBWT) with 4 nacelles

\section{Near-Field Sonic Boom Prediction Methods Available at NASA Before the Workshop}

Early work applied CFD within a fraction of a body length. This very near-body signature was propagated to the wind tunnel measurement locations with ANET. ${ }^{11}$ Cheung, Edwards, and Lawrence ${ }^{12}$ applied UPS3D with ANET to cone cylinder, wing, and delta wing body configurations. Cliff and Thomas ${ }^{13}$ applied FLO60, TEAM, and AIRPLANE with ANET to the same cone cylinder, wing, and delta wing body configurations. Madson $^{14}$ applied TranAir to these same configurations. Cliff et al. ${ }^{10,15}$ applied HFLO4 and AIRPLANE with ANET to a wing body nacelle tail at near-field distances from $1 / 4$ to $1 / 3$ of a body length. Djomehri and Erickson ${ }^{16}$ applied an adaptive unstructured grid FELISA method with ANET to airfoil, cone cylinder, wing, and delta wing body configurations.

Fouladi ${ }^{17}$ applied linear VGRID sources and USM3D to isotropic grids of a body of revolution. Carter and Deere ${ }^{18}$ examined a number of grid sourcing methods in VGRID and the grid adaptation methods ADAPT, ADV, and CRISP. The SSGRID grid modification tool, an a priori adaptation method used in the workshop, was also introduced. Campbell et al. ${ }^{19}$ provides a more detailed description of ADV and SSGRID. Pirzadeh ${ }^{20}$ developed a volume sourcing method that allows for specification of a swept and refined volume region below an aircraft configuration.

Prior to the workshop, FUN3D had an existing anisotropic output adaptation scheme for tetrahedral body-fitted grids. ${ }^{21}$ Lee-Rausch et al. ${ }^{22}$ examined the near-body signatures of cone cylinder and wing body configurations and Jones, Nielsen, and Park ${ }^{23}$ performed detailed validation exercises on cone cylinder geometries. The body-fitted approach to adaptive griding exhibited robustness difficulties near curved surfaces that were alleviated by the cut-cell approach utilized at the workshop.

In early 2008, Nemec, Aftosmis, and Wintzer ${ }^{24}$ used Cart3D along with adjoint-based mesh adaptation to predict the pressure signature of a diamond airfoil. This work used both an off-body functional to drive the adjoint and introduced a mesh alignment technique based on the Mach-angle of the free-stream flow. The capability was built upon a preexisting mesh adaptation scheme ${ }^{25}$ and adjoint solver. ${ }^{26}$ 3D examples using the Cartesian-adjoint approach were presented in June 2008, including many cases similar to those used in the workshop. ${ }^{27}$

\section{Methods}

There are five methods used in this study. Accurately predicting near-field signatures is a challenging task that requires the combination of a flow solver with a specialty grid generation or grid adaptation technique. A signature propagation technique is also employed in conjunction with the flow solver for two methods. A detailed description of each method is provided in the following sections.

\section{III.A. Component Techniques}

The ANET, EASS, and Cart3D techniques are each used by two of the methods, so they will be described first in subsections. AIRPLANE-ANET and Cart3D-ANET use AIRPLANE and Cart3D flow solvers to propagate the signatures less than one body length and transfer the solution to ANET, a signature propagation tool. Cart3D-Adjoint combines the Cart3D flow solver with an adjoint-based adaptation scheme. 
FUN3D-Adjoint utilizes a similar adjoint-based adaptation scheme, but uses a tetrahedral background grid. USM3D-SSGRID is the USM3D flow solver used directly with a priori grid shearing and stretching of a baseline isotropic grid.

\section{III.A.1. ANET}

ANET is a sonic boom extrapolation code originally developed by Thomas. ${ }^{11}$ It utilizes a waveform parameter method ${ }^{28}$ where the signature is completely described by a set of parameters. Equations are obtained for the time rates of change of these parameters and integrated in time to propagate the signature. ANET is typically used with a standard atmospheric model to propagate the near-field signature to the ground. When combined with the AIRPLANE and Cart3D in this article, a uniform atmospheric model is assumed to propagate the signature within the wind tunnel test section.

\section{III.A.2. EASS}

Mesh generation techniques for external flows typically have a fine grid near the model which coarsens away from the model until the outer boundary is reached. Accurate off-body signature prediction requires constant spacing in important propagation regions. This region of constant spacing is described as Elliptical/Annular Swept Sector (EASS) depicted in Fig. 2.

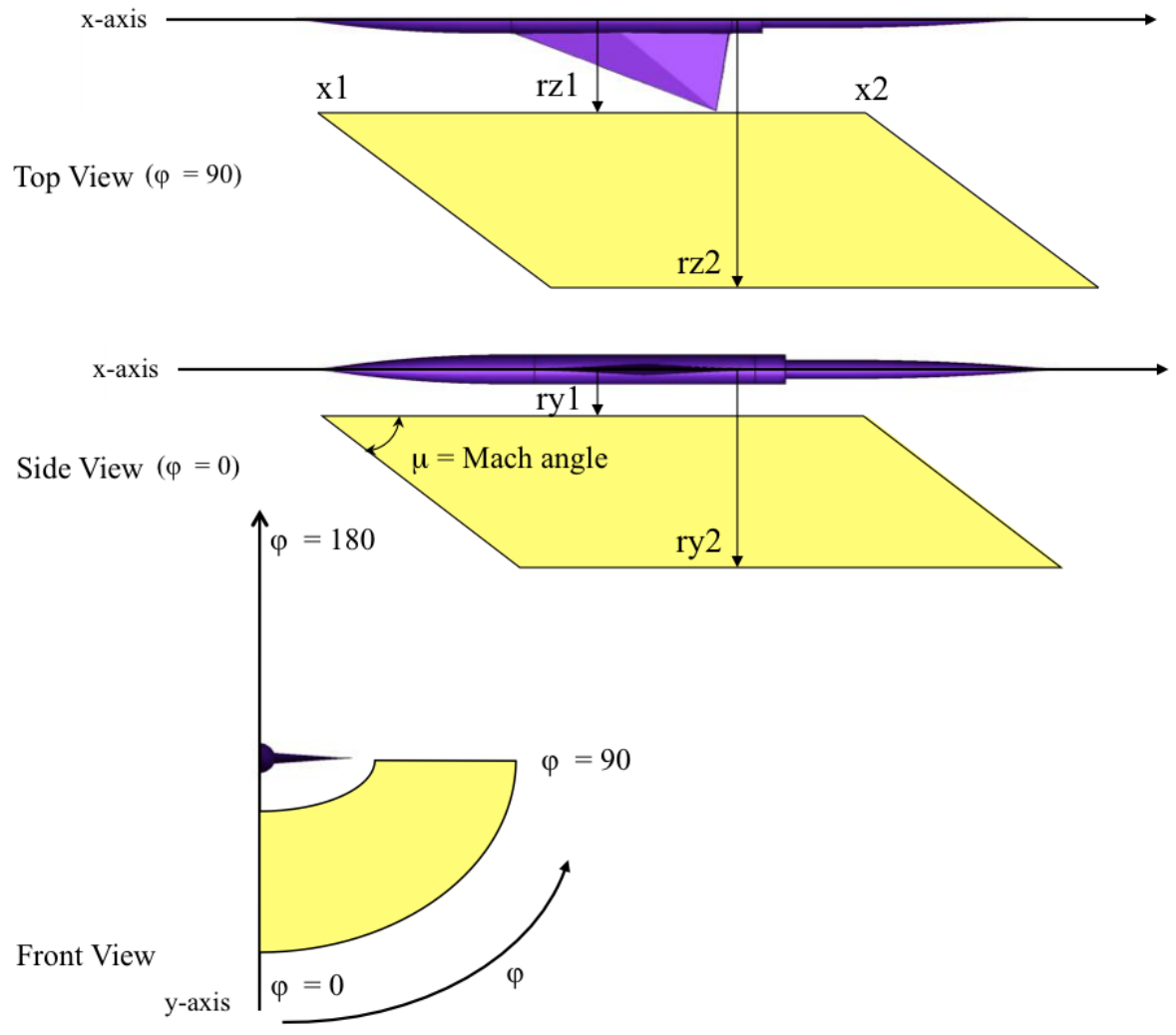

Figure 2. Diagram of EASS refinement region parameterization.

The regions in Fig. 2 are described by providing the Mach number, $x 1, x 2, r y 1, r y 2, r z 1, r z 2$, and off-track angle $\varphi$. The region can be annular when $r y 1$ and $r z 1$ are greater than zero. Most often they are defined to be circular in front view by setting $r y 1=r z 1$ and $r y 2=r z 2$. Elliptically shaped refinement regions can be used when the region of the aircraft is non-circular, such as a winged configuration in axial cross section. Refinement regions or "sectors" are rotated for azimuthal angles from 0 (below the aircraft) to a user prescribed angle $\varphi$ up to 180 degrees. 


\section{III.A.3. Cart3D}

The Cart3D package uses a Cartesian cut-cell approach ${ }^{29}$ in which the governing equations are discretized on a multilevel Cartesian mesh with embedded boundaries. The Cart3D flow solver is combined with the ANET propagation method and an adjoint-based grid adaptation scheme, which are described in the following sections. The mesh consists of regular Cartesian hexahedra everywhere, except for a layer of bodyintersecting boundaries as illustrated in Fig. 3. A finite volume method is applied in the computational domain with a weak imposition of boundary conditions.

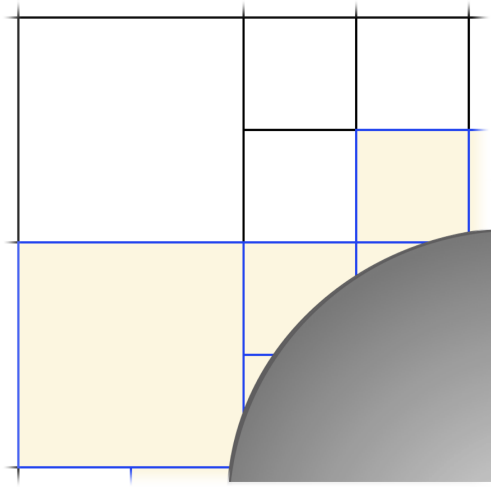

Figure 3. Multilevel Cartesian mesh in two-dimensions with a cut-cell boundary.

Although it consists of nested Cartesian cells, the mesh is viewed as an unstructured collection of control volumes making the approach well-suited for solution-adaptive mesh refinement. The refinement is performed in discrete refinement levels, where a cell is split in the Cartesian directions. Figure 3 illustrates two refinement levels: the large squares on the left and the small squares on the right. Steady-state flow solutions are obtained using a five-stage Runge-Kutta scheme with local time stepping and multigrid. The flux-vector splitting approach of van Leer ${ }^{30}$ is used. Domain decomposition via space-filling curves permits parallel computation; for more details see Aftosmis, Berger, and Murman ${ }^{31,32}$ and Berger, Aftosmis, and Murman. ${ }^{33}$

In boom propagation problems, the Cartesian mesh is rotated to roughly align the mesh cells with the free stream Mach-wave angle. Mesh rotation also permits the cells stretching along the dominant wave propagation direction to directly increase the per-cell signal propagation distance. Details of these techniques are available in Wintzer, Nemec, and Aftosmis. ${ }^{27}$ Nemec, Aftosmis, and Wintzer ${ }^{24}$ demonstrate a saving of 3 in grid size with equivalent accuracy in $2 \mathrm{D}$ due to rotation and anisotropic refinement with aspect ratio of 4. Saving a factor of 16 in $3 \mathrm{D}$ grid size with equivalent accuracy by rotation is documented by Cliff et al. ${ }^{34}$

\section{III.B. AIRPLANE-ANET}

The AIRPLANE-ANET method ${ }^{34}$ is an Euler unstructured-tetrahedra-grid method. ${ }^{35,36}$ The mesh generator in its original formulation provided a smooth gradation from the fine surface mesh to the coarse far field boundaries. This smooth gradation of the volume mesh density is not ideal for sonic boom computations because the off-body mesh coarsens too quickly. To increase the mesh density within the sonic boom region of influence so that the pressure signature can be propagated sufficiently far from the model, the mesh generator was modified to allow a user to refine meshes in specified regions for sonic boom computations. This refinement region is defined with EASS.

Automatic cell refinement based on the edge length and distance from the model is employed. Figure 4 shows three axial slices of the 69-degree delta wing body and sting configuration colored by the maximum edge length, Max E. Conditional splitting based on edge length allows for smoother changes in grid densities and simplifies the process by only requiring one EASS regions.

EASS refinement permits a fairly efficient use of mesh points with increased accuracy for boom assessment provided the aircraft bow and tail shocks stay within the EASS. Pressure signatures were extracted in the very near field at 0.4 body lengths below the configuration and extrapolated to wind tunnel test altitudes with ANET via a uniform atmosphere model. 


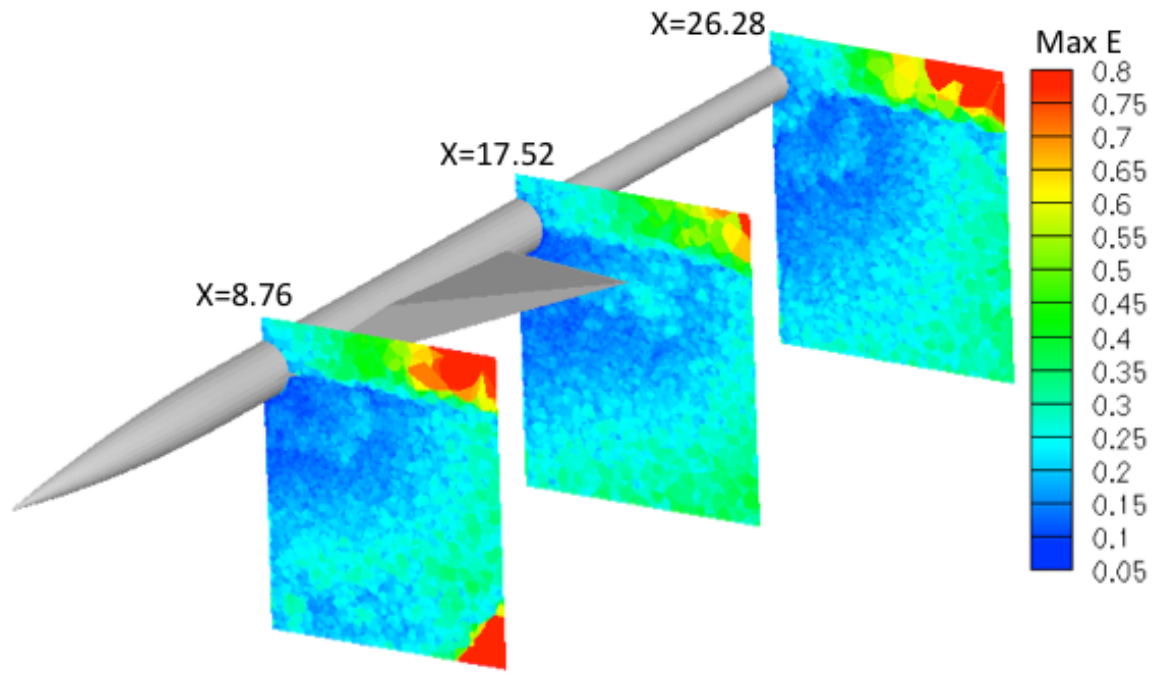

Figure 4. Delta wing body and contours of maximum edge length (Max E) in an EASS region.

\section{III.C. Cart3D-ANET}

In the Cart3D-ANET approach, ${ }^{34}$ the basic Cartesian method is used either with or without mesh rotation and combined with the pre-specified mesh refinement using EASS. The independence of the surface grid is advantageous in sonic boom analysis because the effects of volume grid density can be studied independently of the surface grid density, and the effects of grid variations can be easily assessed.

EASS refinement regions were implemented in Cart3D by the development of a software tool that generates the defining input of a large number of rectangular regions that fill an EASS refinement region. These rectangular regions are provided to Cart3D to trigger adaptive refinement. ${ }^{25}$ The swept refinement region can be accurately prescribed with the large number of small rectangular regions inside the expected zone of influence of the solutions and a single level of Cartesian mesh refinement throughout the EASS region can be easily obtained. This allows for simple grid-refinement studies with Cart3D to compare the differential pressure distribution with mesh refinement level. ${ }^{34}$ The level of refinement shown in Fig. 5 is what was required to obtain accurate pressure signatures near the boundaries of the EASS region. ${ }^{34}$

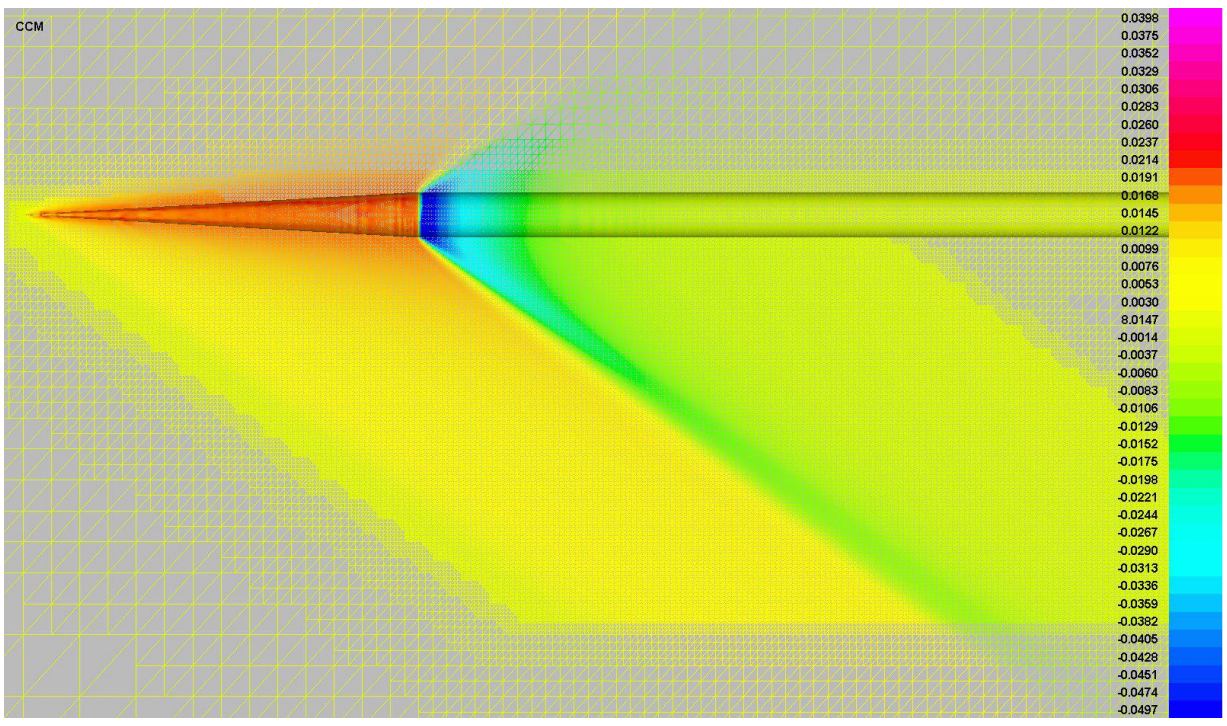

Figure 5. Symmetry plane grid and pressure coefficient with EASS refinement region for the cone-cylinder configuration, Cart3D, $M=1.68, \alpha=0.0$ degrees. 
EASS refinement was also combined with mesh rotation to the dominant propagation direction. This resulted in significant savings in the required computational mesh size for the same level of accuracy as non-rotated cases. Saving a factor of 16 in grid size with equivalent accuracy by rotation is documented by Cliff et al. ${ }^{34}$ Figure 6 shows the same configuration at equivalent accuracy as Fig. 5 with a factor of 16 smaller grid.

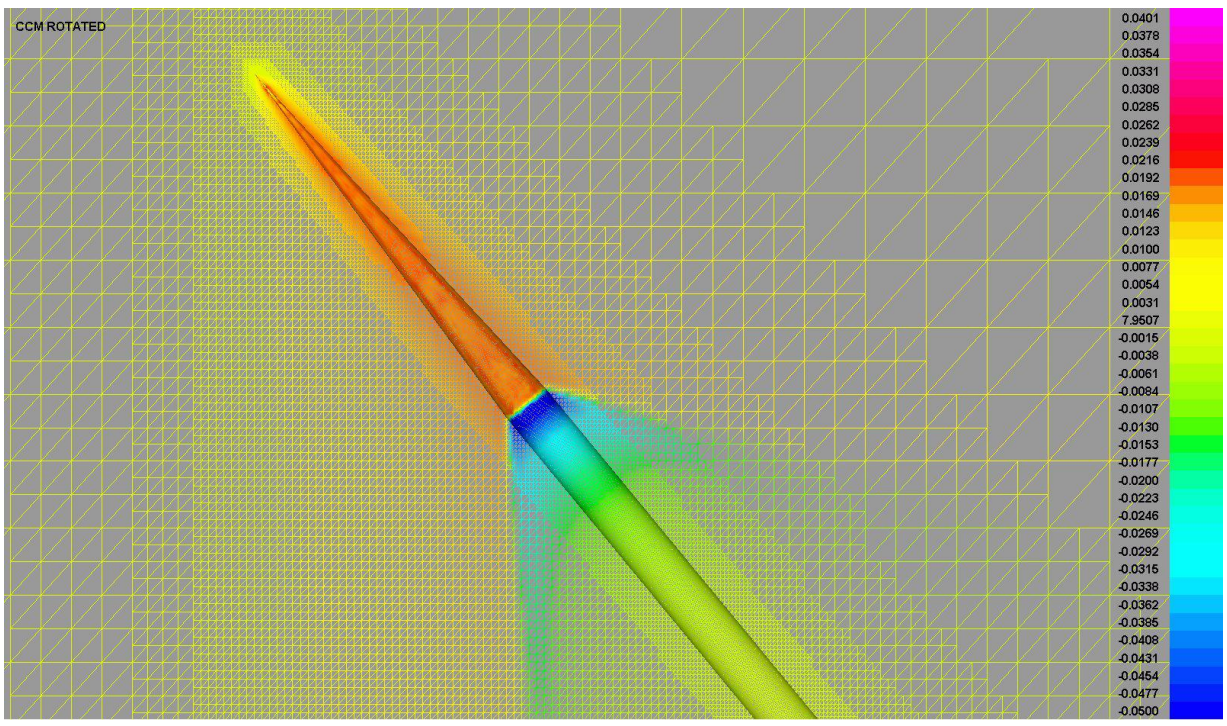

Figure 6. Symmetry plane grid and pressure coefficient with EASS refinement region and configuration rotation for the cone-cylinder configuration, Cart3D, $\mathrm{M}=1.68, \alpha=0.0$ degrees.

\section{III.D. Cart3D-Adjoint}

In 2005, a duality-preserving discrete adjoint approach was introduced for Cart3D by Nemec and Aftosmis ${ }^{26}$ for aerodynamic shape optimization. This adjoint solver shares the same basic data structures, domain decomposition and other infrastructure with the primal solver and achieves similar performance. Nemec and Aftosmis ${ }^{37}$ first applied this method for output error-estimation and adaptive refinement in 2007 using an approach similar to that of Venditti and Darmofal ${ }^{38}$ and others. ${ }^{39-41}$ In 2008, it was applied for boompropagation problems using a quadratic form of the pressure $p$,

$$
\mathcal{J}=\int_{0}^{L}\left(\frac{p-p_{\infty}}{p_{\infty}}\right)^{2} \mathrm{~d} l
$$

where the integration is performed along a sensor of length $L$ placed in the field where the signature is measured.

The adjoint-based error-estimation then tailors the mesh refinement to reduce the error in the pressure signature at the location of the sensor. Error in this signal can be either driven below some pre-specified value, or alternatively, reduced as much as possible using a worst-errors-first strategy for a desired mesh size. Adaptation is performed incrementally by cycling between the primal and adjoint solvers, with no more than one level of cell refinement being performed at a time. With this strategy, typical simulations cost 3-5 times that of a single flow solve on the final mesh. Figure 7 shows a typical mesh produced by this method for flow over an axisymmetric body at 1.6 Mach and 0 degrees angle of attack. The mesh was produced after 11 cycles of adaptive refinement driven by the pressure signature from the shaded portion of the body on an array of four sensors located 1.8 body lengths $h / l=1.8$ away. This verification example from Aftosmis et al. ${ }^{5}$ shows that the pressures along all sensors collapse to the same signature, as expected in axisymmetric flow.

\section{III.E. FUN3D-Adjoint}

The FUN3D-Adjoint method is an output-adaptive cut-cell method utilizing tetrahedral background grids. The complete adaptive process is documented by Park. ${ }^{4}$ The cut-cell flow solver including gradient recon- 

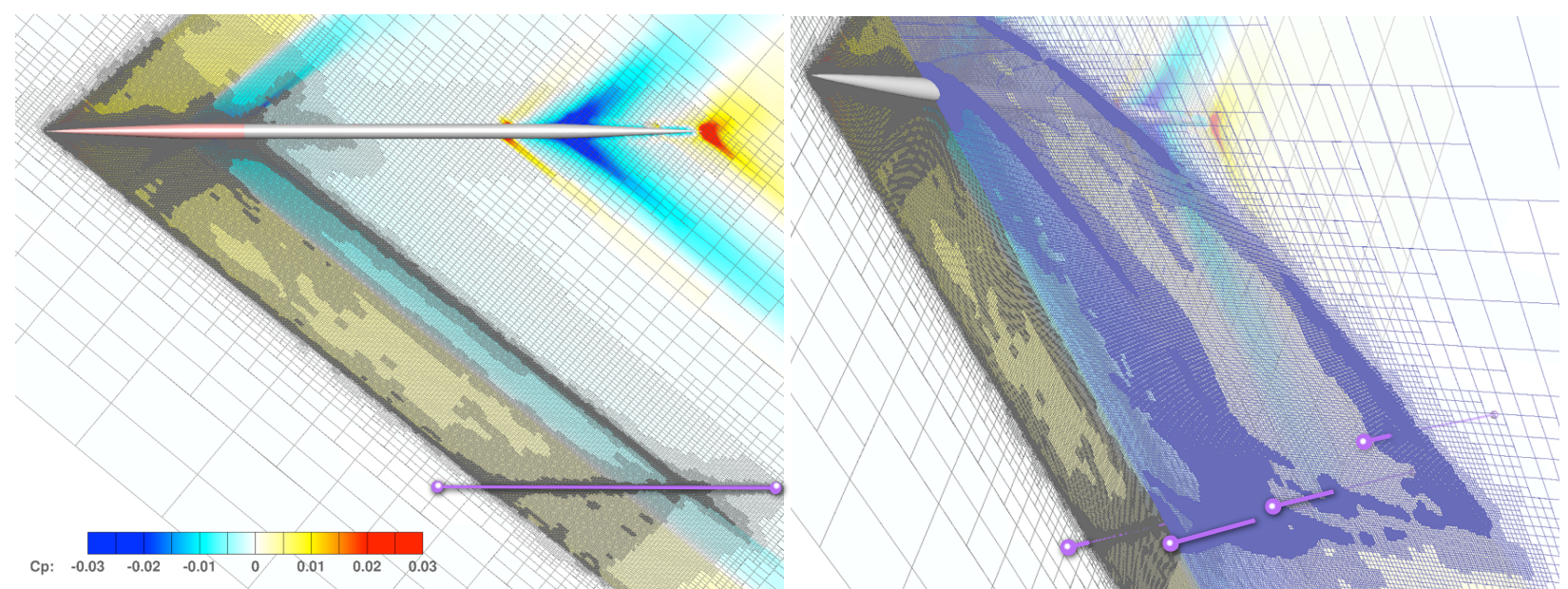

Figure 7. Typical mesh and pressure for the Cart3D-Adjoint method taken from the axisymmetric example at 1.6 Mach and 0 degrees angle of attack. ${ }^{5}$ Adaptation driven by sensors are located at $0,15,30$, and 45 degrees off centerline with $h / l=1.8$. The final mesh contains approximately 13M cells.

struction and limiting is summarized by Park and Darmofal ${ }^{42}$ and the parallel grid mechanics are summarized by Park and Darmofal. ${ }^{43}$

A triangulated surface mesh of the geometry is Boolean subtracted from the median duals of the background grid to form the computational domain. To introduce the 3D cut-cell method a simple 2D example is presented. The primal triangular grid is shown in Fig. 8(a). The control volumes used by the flow solver are the median duals of this triangular mesh in Fig. 8(b). These median duals are constructed by gathering the three dual faces that are inside each primal triangle. These faces connect the center of the triangle to the midpoints of its edges. The geometry in this example is a diamond airfoil, shown with the uncut median dual background grid in Fig. 8(c). The airfoil geometry is Boolean subtracted from this background grid removing the portion of the background grid that is external to the flow domain, Fig. 8(d). This is the same operation that Cart3D performs, but here the background grid is tetrahedral instead of Cartesian. The result of the Boolean subtraction are the control volumes used by the flow and adjoint solver.

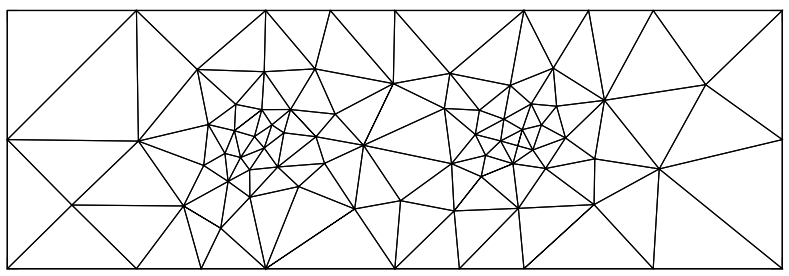

(a) Primal grid.

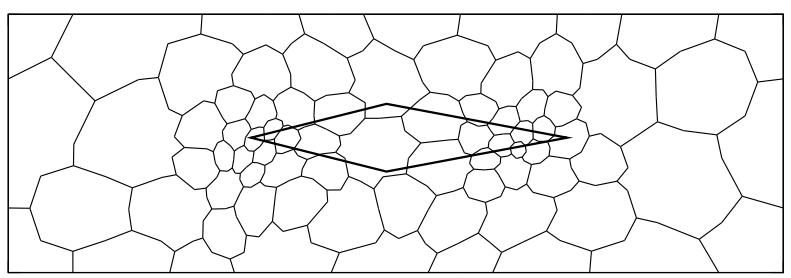

(c) Median dual grid with geometry.

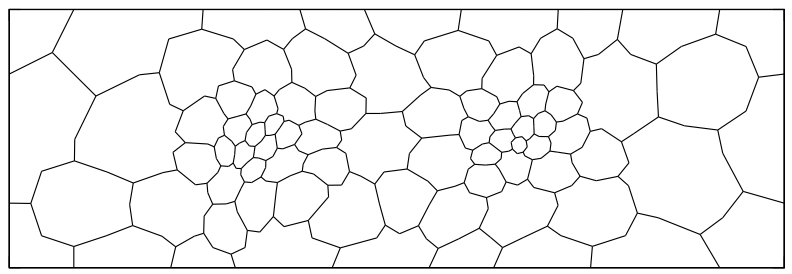

(b) Median dual grid.

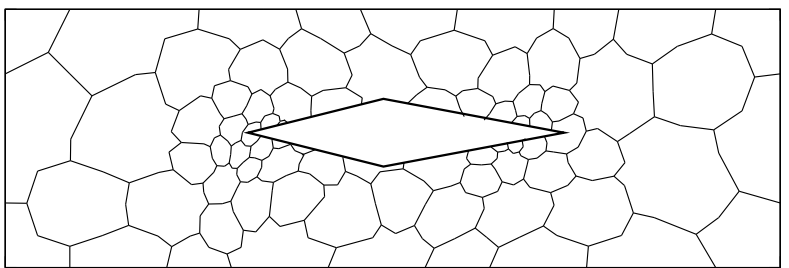

(d) Resulting cut-cell grid.

Figure 8. Cut cell illustration of a diamond airfoil in $2 \mathrm{D}$ with a simplex background grid.

Weighted least-squares reconstruction is performed to increase the accuracy of the scheme. These reconstructed gradients are reduced with a continuously differentiable heuristic limiter that permits good iterative convergence. ${ }^{42}$ Backward facing steps, e.g., blunt trailing edges, can create strong expansions in Euler flows. These strong expansions can create difficulties for approximate Riemann solvers. A transpiration boundary condition is applied to boundary faces with downstream pointing normals to lessen the strength of these 
expansions while retaining some of the characteristics of wake flows. This boundary condition applies free stream velocity state, $u_{\infty}, v_{\infty}$, and $w_{\infty}$ with a density and pressure of $\rho=0.3 \rho_{\infty}$ and $p=0.3 p_{\infty} .{ }^{4}$

An exact discrete adjoint solution is computed based on a pressure $p$ based sonic boom sensor cost function,

$$
\mathcal{J}=\iint_{s}\left(\frac{p-p_{\infty}}{p_{\infty}}\right)^{2} \mathrm{~d} s,
$$

integrated over a cylindrical surface $s$ centered at the model that passes through the wind tunnel measurement locations. The primal and adjoint solution are reconstructed on the grid with two methods. These different reconstructions are employed to form an adaptive indicator. ${ }^{4}$ This adaptive indicator is combined with the Mach Hessian to compute the requested metric for the background grid, see Venditti and Darmofal. ${ }^{44} \mathrm{~A}$ parallel grid adaptation scheme $e^{4,43}$ is applied to the background grid to produce a new background grid based on the requested metric. This adaptive process is applied multiple times until the resolved pressure signature at the sensor location is available. The initial and final symmetry plane grid of an axisymmetric configuration $^{45}$ are shown in Fig. 9 to illustrate this process. The extremely coarse isotropic initial grid, Fig. 9(a), is created independently of Mach number or angle of attack. There is no attempt to represent the propagation of the shocks to the sensor location, only the geometry in the upper right is partially resolved. After 17 adaptation cycles, the final grid, Fig. 9(b), accurately resolves the signature at $h / l=6$.

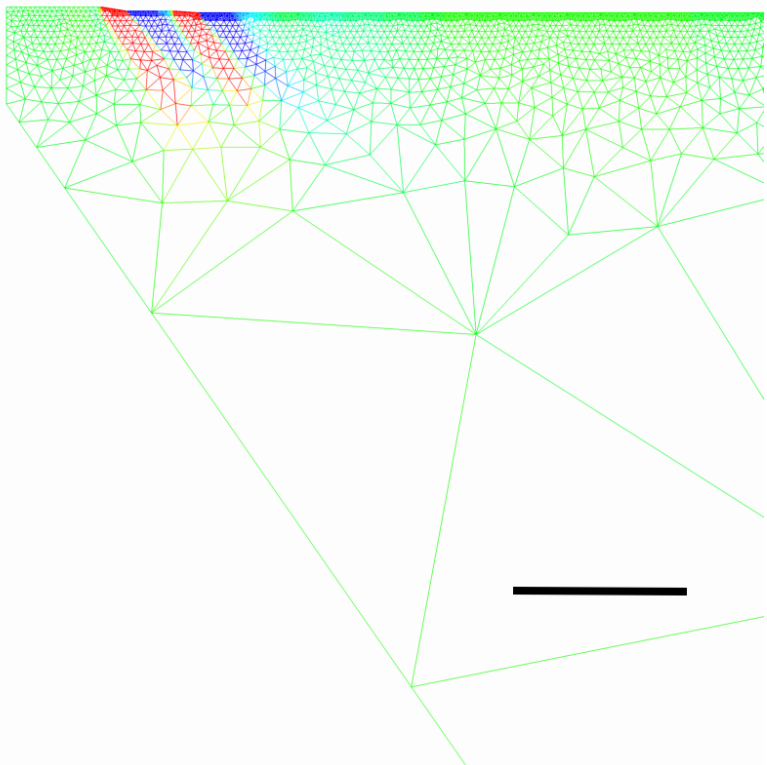

(a) Initial symmetry plane grid.

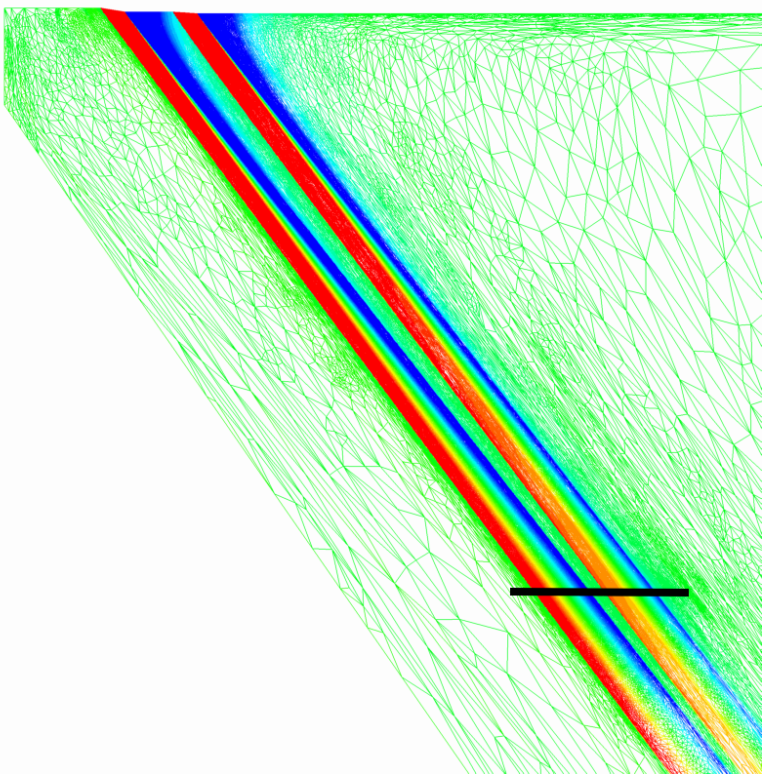

(b) Adapted symmetry plane grid.

Figure 9. Symmetry plane grid colored with pressure for an axisymmetric configuration FUN3D-Adjoint simulation. ${ }^{45}$ The intersection of the sensor surface with the symmetry plane is shown in black.

The use of cut-cells with an output-based adaptive scheme automates the volume grid generation task after the triangular surface mesh is generated. This robust adaptation scheme allows extremely coarse isotropic initial grids that can be generated without a priori knowledge of shock locations or Mach angles. The general anisotropy of the adapted background grids allows for accurate center line and off-center line signal prediction. ${ }^{42}$

\section{III.F. USM3D-SSGRID}

The TetrUSS CFD software package ${ }^{46}$ includes the VGRID grid generation code ${ }^{47}$ along with the USM3D Navier-Stokes flow solver. ${ }^{48}$ The VGRID code uses a combination of the advancing layer and advancing front methods to fill the computational domain with tetrahedral cells. The grid spacing on the configuration surfaces, outer boundaries, domain interior is related to the strength of sources placed in the domain. While point and line sources can be used to control field grid density, the resulting grid density can be nonuniform. A more recently developed source type, the volume source, ${ }^{20}$ allows much better control of field grid spacing and is discussed further in the following section. 
The USM3D code is a cell-centered, finite-volume Navier-Stokes flow solver that uses Roe flux-difference splitting ${ }^{49}$ to compute inviscid flux quantities across the faces of the tetrahedral cells. The parallel version of the flow solver was run inviscidly using the implicit mode for the cases presented in this study. The minmod limiter was typically used to ensure numerical stability during the initial iterations, and was then turned off for the final cycles to minimize dissipation in the sonic boom signature.

\section{III.F.1. Automated Source Placement for Grid Generation}

The geometry is defined with GRIDTOOL. ${ }^{50}$ VGRID requires a specification of the requested grid density in the form of sources ${ }^{20,51}$ to convert this geometry definition into a grid. AUTOSRC is developed to provide an automated, knowledge-based approach to the placement and sizing of these VGRID sources. AUTOSRC identifies key geometry curves for the patches associated with components such as the fuselage, wing, winglet, nacelles, pylons, canard, and horizontal and vertical tails, then uses this information to determine line source location and grid spacing size.

Figure 10 illustrates how the sources (yellow lines) are placed along the leading and trailing edges of lifting surfaces and down the centerline of the fuselage. The source layout for nacelles includes a ring of line sources around the inlet and exit, as well as a stream-wise source down the centerline of the nacelle.

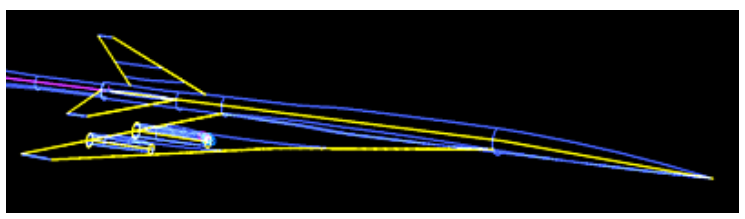

Figure 10. VGRID line sources (in yellow) automatically generated by AUTOSRC.

In addition to the automated layout of line sources described above, two options that use the new volume source capability in VGRID ${ }^{20}$ have been included in AUTOSRC. The first option is used for the computation of the near-to-mid field sonic boom signature of an aircraft and involves placing a vertical-axis cylindrical volume source just below the fuselage. This volume source is automatically sized and located under the configuration to provide a dense field grid for resolving shocks and expansions (see the yellow cylinder around the dashed center line in Fig. 11). This grid is further modified by the SSGRID code, which is described in the next subsection, to help reduce dissipation in the flow solution. The second option places a horizontal-axis cylindrical source behind the nacelle exit to create a denser grid to resolve the engine plume shape for powered simulations (see the magenta cylinder in Fig. 11). Currently, the length of the plume source is a user input, with a recommended value of three nacelle lengths based on limited experience. Further details on the AUTOSRC method can be found in Li et al. ${ }^{52}$

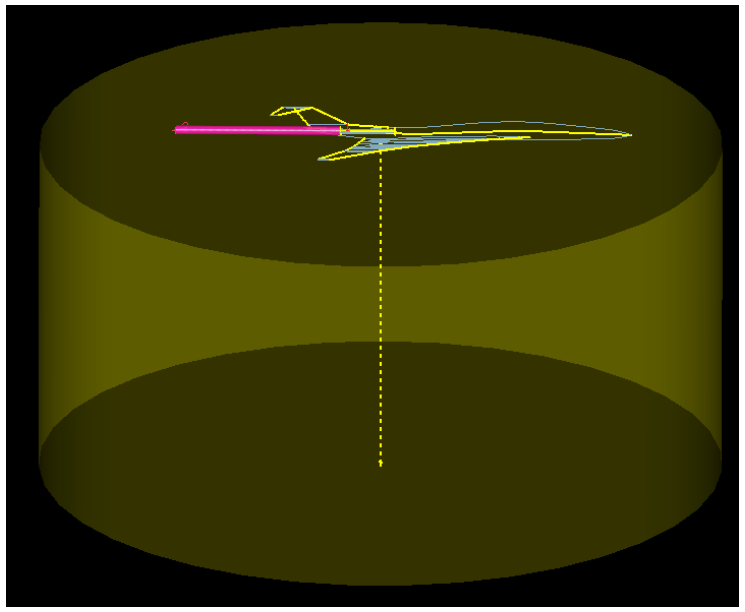

Figure 11. VGRID line and volume sources for boom analysis, including plume effects. 


\section{III.F.2. Stretching and Shearing of the Field Grid}

As mentioned in the previous section, the initial grid is typically generated with VGRID using fairly large grid spacing away from the body, except for a region of dense grid underneath the vehicle that is created using a cylindrical volume source. The left side of Fig. 12 shows the grid generated for the symmetry plane using this technique. While this approach does improve the propagation of flow features in the dense zone relative to outer coarse grid regions, it significantly increases the number of grid cells and is thus not practical for use in reaching the typical mid-field location of 3-5 body lengths below the aircraft. In general, the cell faces are not aligned with the flow features, which are dissipated as they pass through the skewed faces.

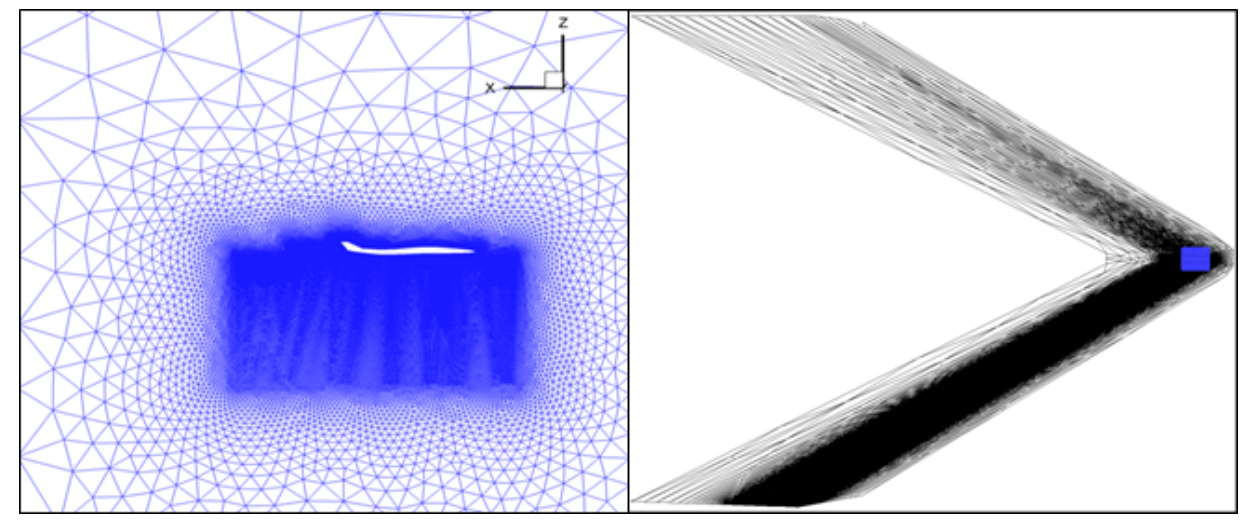

Figure 12. Original (left) and final stretched/sheared symmetry plane grid from SSGRID.

The SSGRID code was developed to address these issues by shearing and stretching the original grid along Mach lines. This process allows fewer grid points to be used to reach a given signature location and aligns the cell faces with the flow features to reduce dissipation. As shearing the grid close to the body might cause it to intersect the wings or other components, an inner cylinder parallel to the body axis and just outside the wing tip is defined within which no grid modification occurs, see Fig. 13. This region tends to be

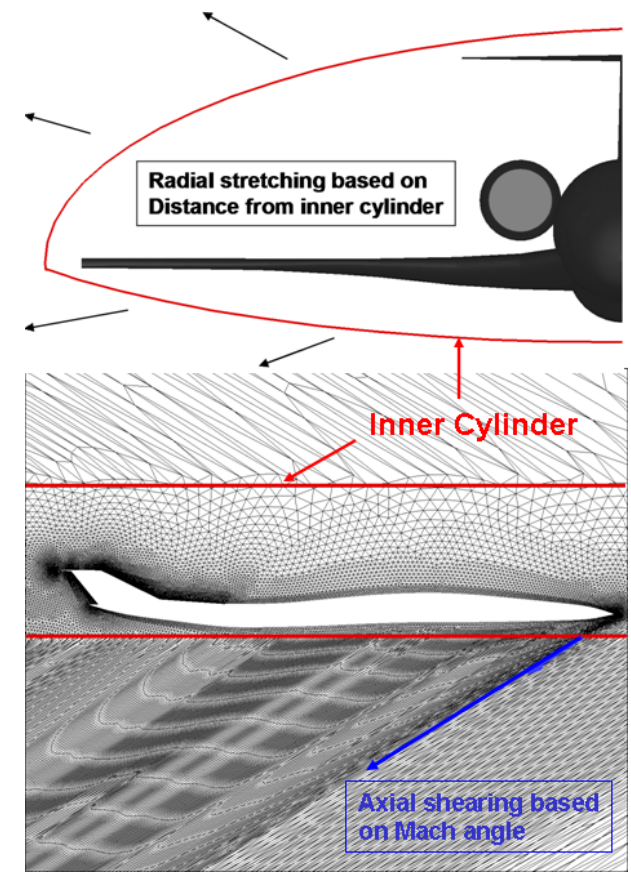

Figure 13. Grid stretching and shearing controlled by an inner cylinder.

much farther from the keel line of the aircraft than it needs to be and could allow unnecessary dissipation to occur before the sheared grid is reached. To remedy this, a variable inner cylinder radius is used based on the keel line. The keel line, primary inner cylinder radius, and variable radius are all automatically 
determined in SSGRID based on the aircraft geometry and initial grid characteristics. The right side of Fig. 12 illustrates the extent of a typical stretched and skewed grid, with the small blue region corresponding to the grid portion shown in the lower half of Fig. 13. The above figures are from Li et al. ${ }^{52}$ where a more detailed description of SSGRID can be found.

\section{Test Cases and Results}

The methods were examined by analysis of five configurations ranging from simple axisymmetric models to lifting models with fuselage, wing, tail, and nacelles, see Table 2. All the analyses for the workshop assumed inviscid flow. The primary focus of the workshop was code-to-code comparisons. Wind tunnel measurements are provided for reference, but there are known differences in the wind tunnel test and simulation that are expected to prevent a perfect match of measured and predicted results.

The analyses assumed steady uniform flow. However, wind tunnels have varying degrees of flow nonuniformities (spatial and temporal fluctuations in Mach number, static pressure, and humidity) ${ }^{53-55}$ and the models are subject to aeroelastic effects since they are slender and have thin wing sections. This vibration can cause a 10-20\% variation in normal force, which is used to infer angle of attack. The flow non-uniformities also smear shocks, increasing their measured thickness. ${ }^{56}$ The reference pressure probe is in a different location in the tunnel than the measurement probe, which is an additional source of uncertainty.

The viscosity effects in the measured data are not captured by the Euler computations. An effect of the boundary layer and boundary layer tripping on the measured near-field signatures has been observed by Wayman et al. ${ }^{57}$ The wind tunnel model base and sting geometry is not documented in the references for the lifting cases. Also, manufactured model geometry may differ slightly from the definitions used in analysis.

Due to slight variations in wind tunnel Mach number, the computed signatures in the following section are shifted slightly in $x / l$ to facilitate comparison. A single obvious feature is selected for each signature and the computed signatures are shifted in $x / l$ until the location of this feature is aligned with the wind tunnel measurements. A uniform expansion is an ideal feature, but a shock location was selected for the lifting cases, which lack a uniform expansion.

\section{IV.A. Cone Cylinder}

The 6.48 degree Cone Cylinder is denoted as Model I(a) in Mendoza and Hicks. ${ }^{7}$ The Mach number is 1.68 and the angle of attack is zero. The signature was measured at an altitude of 86 inches, which is at 10 body lengths. The body is defined as the cone in Fig. 14, which has a 8.6 inch reference length. This configuration was chosen because it represents a near-field pressure signature with finite rise time.

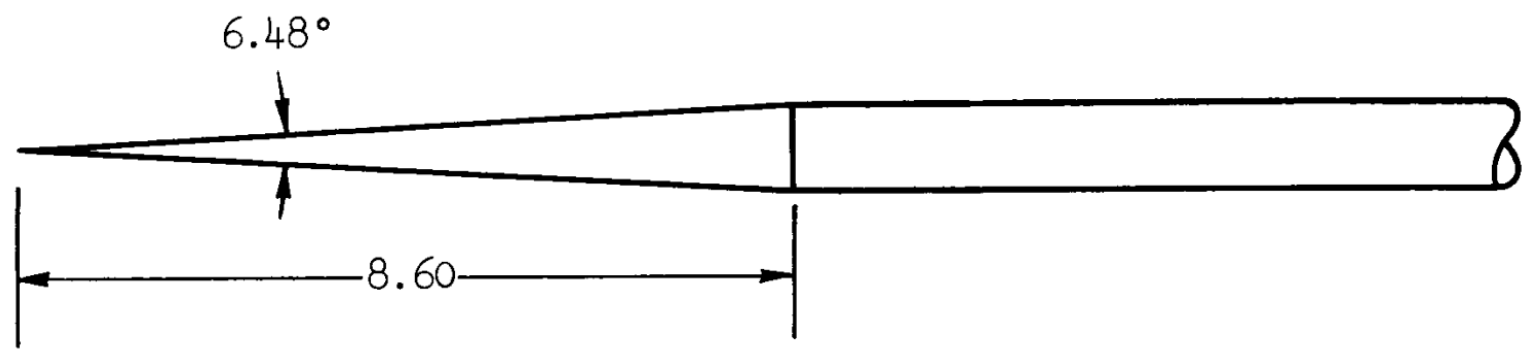

Figure 14. Cone Cylinder geometry (dimensions in degrees and inches). ${ }^{7}$ The model is defined as the cone.

The signatures from all methods are compared with experimental measurements digitized from the report,${ }^{7}$ which are the filled circles in Fig. 15. The vertical axis dP is the normalized disturbance pressure, $\left(p-p_{\infty}\right) / p_{\infty}$. The horizontal axis is $\mathrm{X}$ normalized by the model length $\mathrm{L}$. The signatures have been shifted slightly in $x / l$ to match pressure at the center of the expansion at $x / l=1.1$ because the location of this expansion is an obvious feature of all the available signatures. The methods involving ANET propagation have lower slopes for the initial compression and shoulder expansion where the cone intersects the cylinder. Other methods are very difficult to distinguish, except for the details of undershoot at $x / l=1.3$. FUN3D-Adjoint has the lowest pressure at the end of the expansion and agrees with Cart3D-Adjoint and USM3D-SSGRID on the location of lowest pressure. The methods that utilize ANET predict the most forward maximum pressure location and most aft minimum pressure location. 


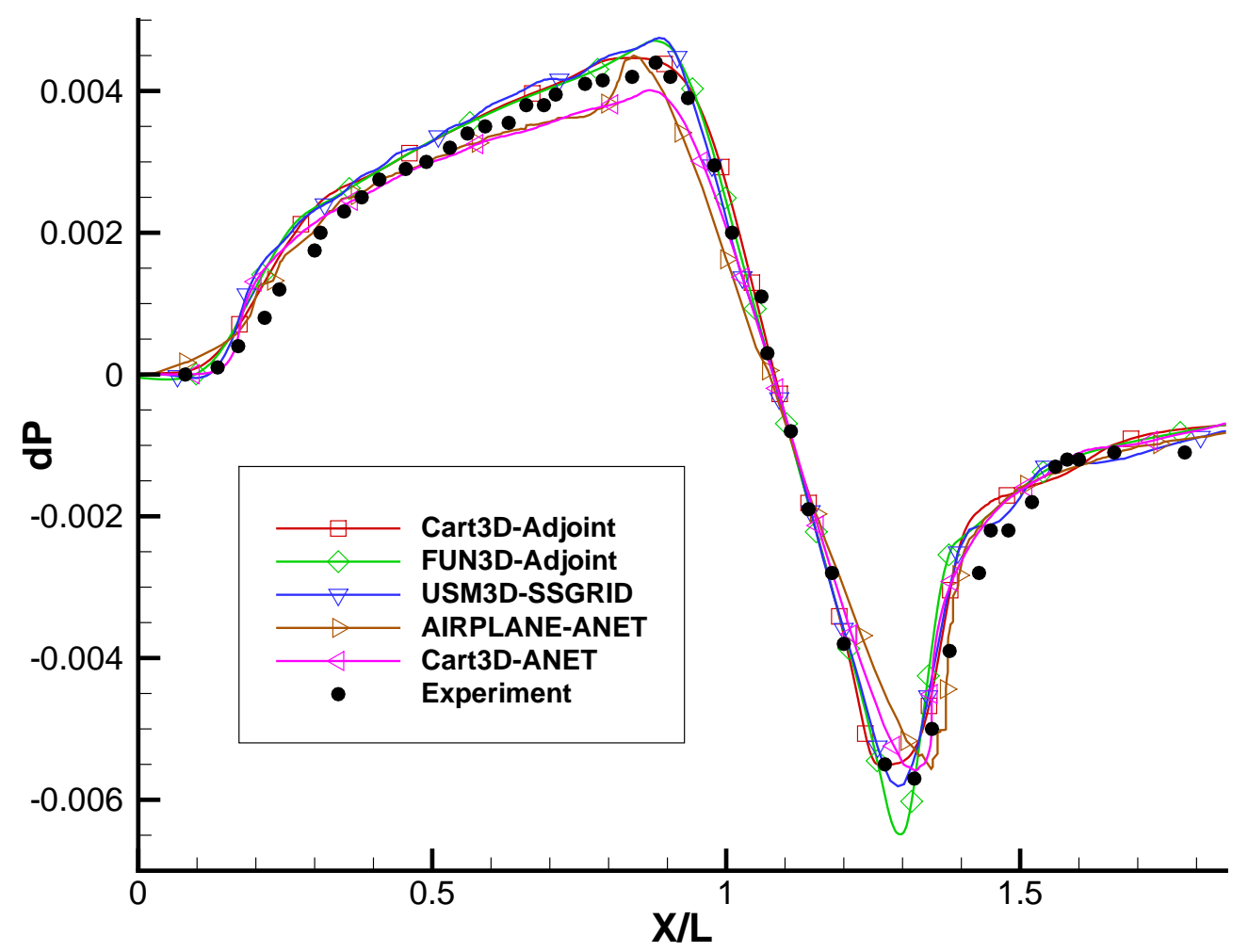

Figure 15. Nondimensional pressure signature for the cone cylinder geometry at $h / l=10.0$. 
This cone cylinder configuration is considered a good configuration for benchmarking simulation. Cone cylinder results were published before the workshop by a number of researchers providing a good history of how this field of prediction has progressed. Cheung, Thomas, and Lawrence ${ }^{12}$ applied UPS3D. Cliff and Thomas $^{13}$ applied TEAM and AIRPLANE to 1.1 and 0.1 body lengths and propagated these near-field signatures with ANET to the wind tunnel measurements at 10 body lengths. Madson ${ }^{14}$ applied TranAir to 0.25 body lengths and propagated these near-field signatures with ANET to the wind tunnel measurements at 10 and 20 body lengths. Djomehri and Erickson ${ }^{16}$ applied an adaptive grid FELISA method to 0.4 body lengths, which was propagated with ANET to 10 body lengths.

\section{IV.B. Parabolic}

The Parabolic body of revolution is denoted as Model 4 in Carlson, Mack, and Morris. ${ }^{8}$ The radius $r$ of the model as a function of $x$ is

$$
r(x)=\sqrt{\frac{0.02}{\pi} x}
$$

see Fig. 16. The Mach number is 1.41 and the angle of attack is zero. The signature was measured at an altitude of 20 inches, which is 10 body lengths of the 2.0 inch model. This configuration was chosen because it is representative of the nose of a typical supersonic transport design.

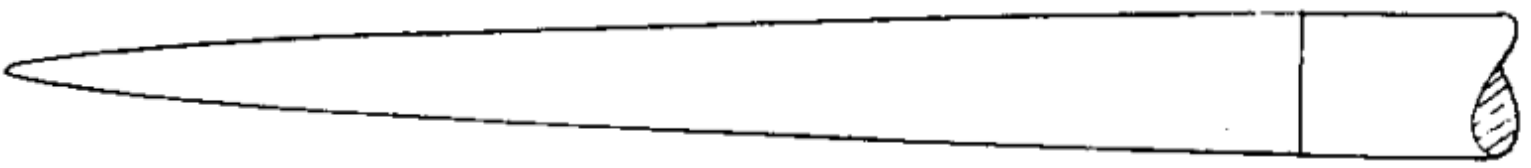

Figure 16. Parabolic Body of Revolution geometry. ${ }^{8}$ The model is defined as the nose to shoulder, which is 2 inch long.

The signatures from all methods are compared with experimental measurements digitized from the report, ${ }^{8}$ which are the filled circles in Fig. 17. The signatures have been shifted slightly in $x / l$ to match pressure at the center of the expansion at $x / l=0.03$. The source of the oscillation of the wind tunnel measurements in the expansion region, $-1.0<x / l<0.0$, is unknown, but oscillations in geometry, reference pressure, static pressure, or Mach number are possible. The methods involving ANET propagation have a lower value of pressure in the parabolic expansion region, $-1.0<x / l<0.0$, and a lower slope in the shoulder expansion at $x / l=0.1$ where the parabolic intersects the cylinder. The USM3D-SSGRID results have slight high frequency waviness in the parabolic expansion region, $-1.0<x / l<0.0$. AIRPLANE-ANET predicts non-zero delta pressure in the ambient flow upstream of the nose shock likely due to dissipation.

\section{IV.C. Quartic}

The Quartic body of revolution is denoted as Model 5 in Carlson, Mack, and Morris. ${ }^{8}$ The radius $r$ of the model as a function of $x$ is

$$
r(x)=x^{\frac{1}{4}} \sqrt{\frac{0.04}{\pi \sqrt{2}}},
$$

see Fig. 18. The Mach number is 1.41 and the angle of attack is zero. The signature was measured at an altitude of 20 inches, which is at 10 body lengths of the 2.0 inch model. This configuration was chosen because it has a strong bow shock and low boom shape.

The signatures from all methods are compared with experimental measurements digitized from the report ${ }^{8}$ which are the filled circles in Fig. 19. The signatures have been shifted slightly in $x / l$ to match pressure at the center of the expansion at $x / l=0.0$. As with the parabolic shape, the source of the oscillation of the wind tunnel measurements in the quartic expansion region $-0.75<x / l<0.0$ is unknown, but oscillations in geometry, reference pressure, static pressure, or Mach number are possible. All the methods have very similar bow shock locations and predict the angle of expansion from the maximum overpressure, which implies that they would propagate equivalently to the ground. The methods involving ANET and USM3D-SSGRID have a slight high frequency oscillation for $-0.75<x / l<0.0$. 


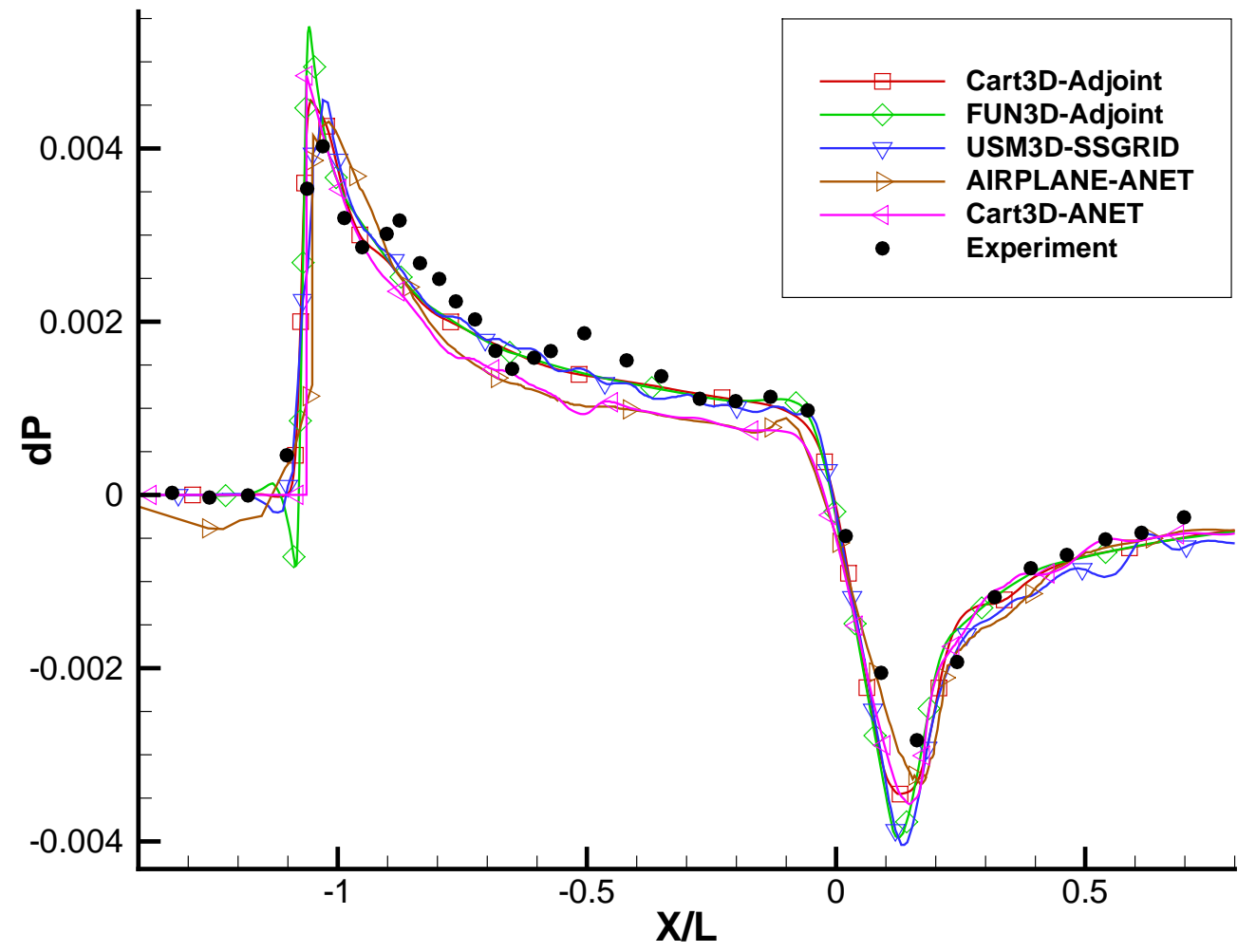

Figure 17. Nondimensional pressure signature for the parabolic geometry at $h / l=10.0$.

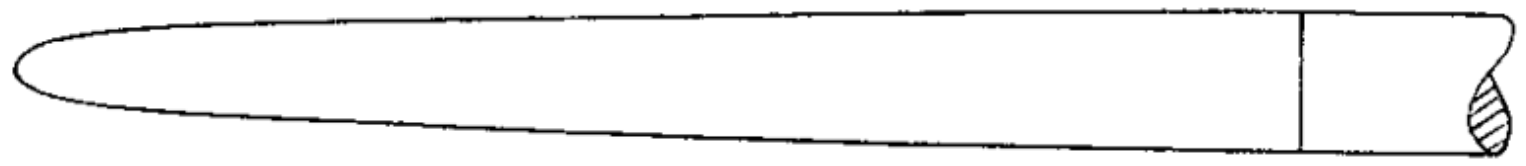

Figure 18. Quartic Body of Revolution geometry. ${ }^{8}$ The model is defined as the nose to shoulder, which is 2 inch long. 


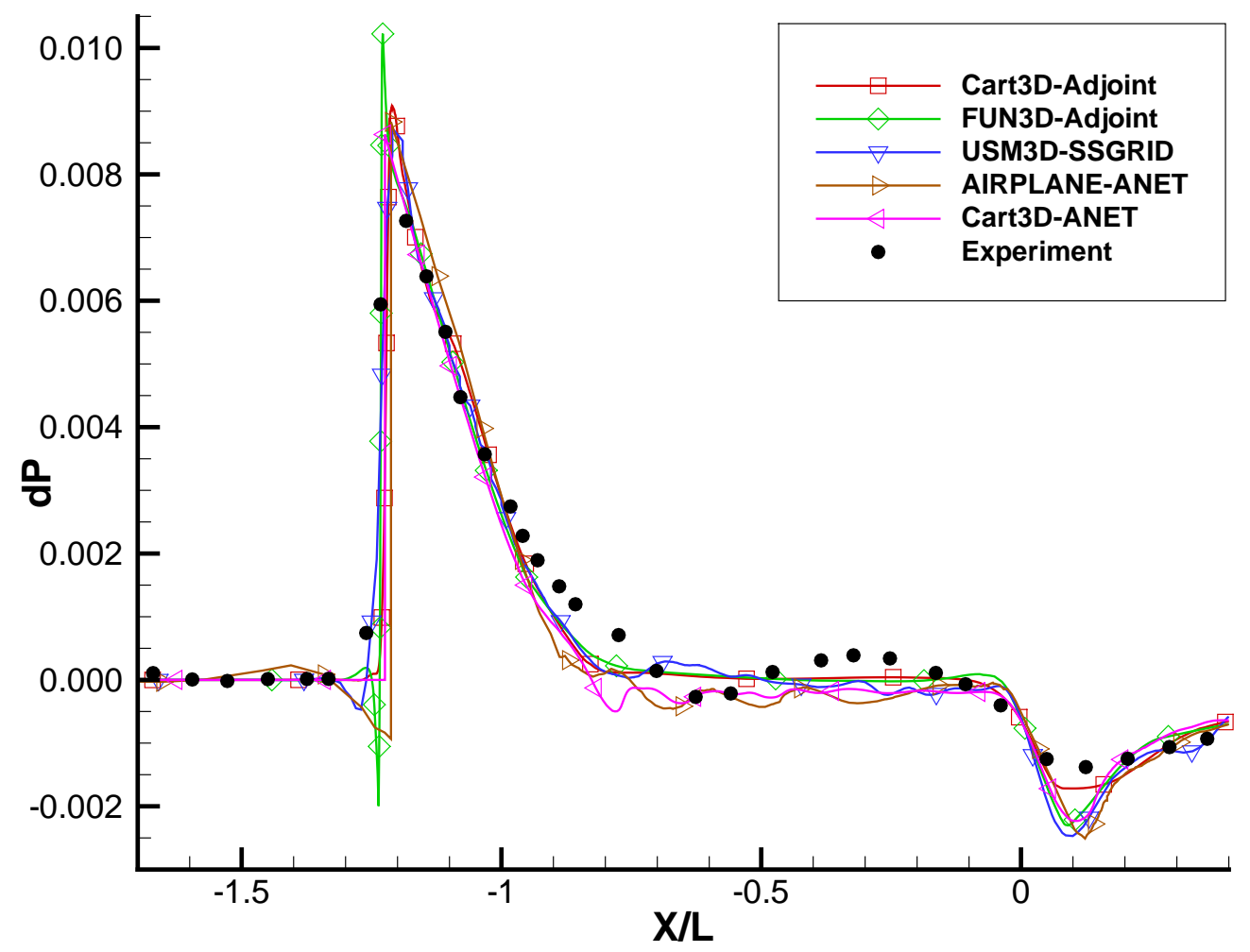

Figure 19. Nondimensional pressure signature for the quartic geometry at $h / l=10.0$. 


\section{IV.D. 69-degree Swept Delta-Wing-Body}

The delta wing body is denoted as Model 4 in a series of sonic boom tests of models with different planforms in the report by Hunton, Hicks, and Mendoza. ${ }^{9}$ The wing thickness to chord ratio is 0.05 and the leading edge sweep is 69 degree, see Fig. 20. The Mach number is 1.68 and the angle of attack is 4.74 degree to correspond to the data measurement with a lift coefficient of 0.15 . The signature was measured at an altitude of $63 \mathrm{~cm}$, which is at 3.6 body lengths of the $17.52 \mathrm{~cm}$ model, see Fig. 20. This configuration was chosen because it is a simple lifting geometry.

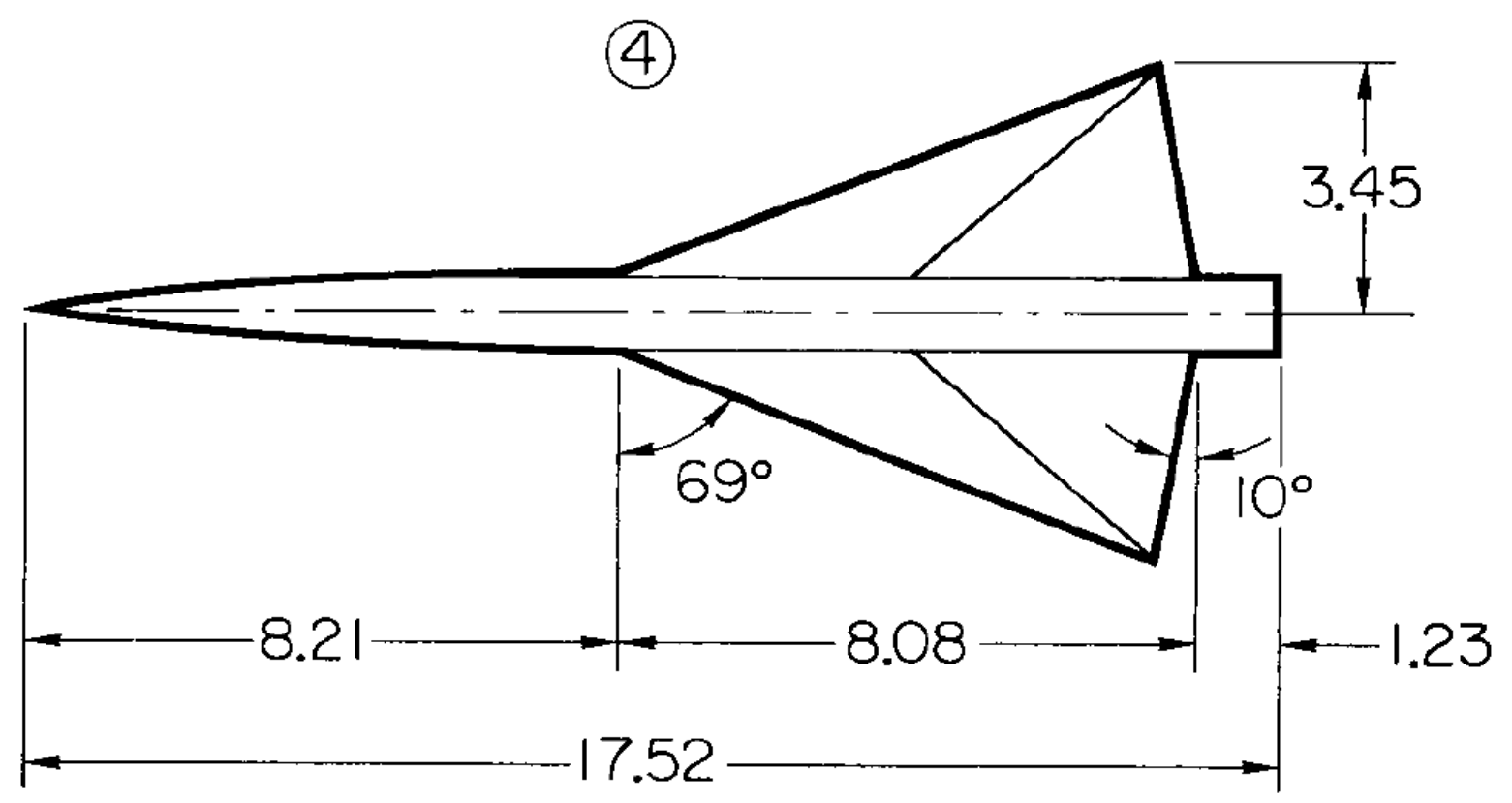

Figure 20. Delta wing body geometry, in $\mathrm{cm}$ and degrees. ${ }^{9}$ The body length is defined as $17.52 \mathrm{~cm}$.

The sting from the original test ${ }^{9}$ is unknown and it was modeled differently by the participants. Cart3DAdjoint used a 90 degree step to reduce the diameter of the fuselage to the smaller sting. All other participants used a 12 degree cone section to reduce the radius without introducing a backward facing step or exceeding the maximum turning angle of the flow. The sensitivity of the aft signature to sting geometry was studied by Cliff and Thomas, ${ }^{13}$ where the 12 degree cone sting produced a higher pressure recovery than the 90 degree step.

The wind tunnel measurements ${ }^{9}$ were only available at 3.6 body lengths. Comparisons of the computational methods was also performed at the intermediate locations of $0.2,0.4,0.8,1.2,2.0$, and 2.8 body lengths, see Fig. 21. Data extracted at these intermediate locations facilitated comparisons of the methods and illustrated how the signature attenuates with distance in the near-field region. The direct extractions of the AIRPLANE and Cart3D computed flow field without the use of ANET are shown for the intermediate locations. The more forward final shock location and higher final pressure recovery for Cart3D-Adjoint is likely due to the use of a 90 degree step to reduce the diameter of the fuselage to the smaller sting.

The closest signature to the model was extracted at $h / l=0.2$, shown in Fig. 22 . This signature lacks a simple expansion, so the signatures have been shifted slightly in $x / l$ to match pressure at the second compression. USM3D-SSGRID shows a slight oscillation in the nose expansion, $0.05<x / l<0.4$. The magnitude of this oscillation reduces at further distances. Cart3D-Adjoint has the highest sting pressure recovery, $1.0<x / l$. This higher pressure recovery moves the last shock forward in Fig. 23 at a $h / l=0.4$ and further distances. Cart3D-Adjoint, FUN3D-Adjoint, and USM3D-SSGRID compare favorably with each other and the wind tunnel measurements ahead of the last shock. AIRPLANE is showing shock thickening at $x / l=0.4$ and $x / l=1.0$, which increases at $h / l=0.8$, Fig. 24 , and $h / l=1.2$, Fig. 25. AIRPLANE results are not presented for $h / l=2.0$, Fig. 26, and $h / l=2.8$, Fig. 27 , because the signature has become too diffused. 


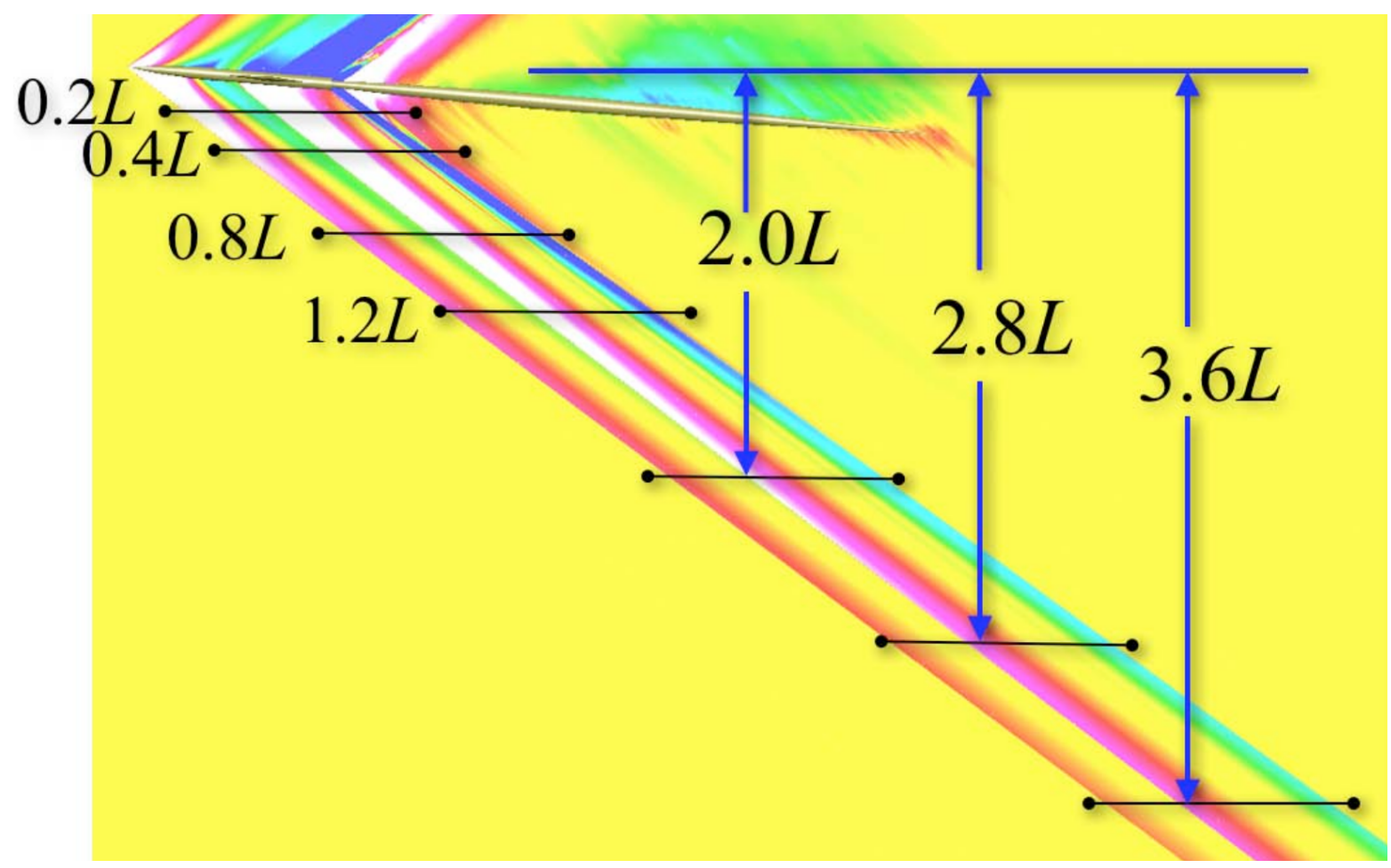

Figure 21. Delta wing body signature extraction locations.

The signatures from all methods are compared with experimental measurements digitized from the report, ${ }^{9}$ which are the filled circles in Fig. 28. AIRPLANE-ANET and Cart3D-ANET use the ANET propagation tool for the signatures at 3.6 body lengths. The ANET methods have a higher pressure for $0.9<x / l<1.2$ The other three methods have very good agreement until the final shock at $x / l=1.2$, which may be due to different sting treatments.

This delta wing body configuration has been used by a large number of researchers as a validation case. Cheung, Thomas, and Lawrence ${ }^{12}$ applied UPS3D. Cliff and Thomas ${ }^{13}$ applied TEAM and AIRPLANE to 0.3 and 0.25 body lengths and propagated these near-field signatures with ANET to the wind tunnel measurements at 3.6 body lengths. Madson ${ }^{14}$ studied various TranAir signature extraction distances and propagated these near-field signatures with ANET to the wind tunnel measurements at 3.6 body lengths. Djomehri and Erickson ${ }^{16}$ applied an adaptive grid FELISA method to 0.3 body lengths, which was propagated with ANET to the wind tunnel measurement locations. Kandil et al. ${ }^{58}$ used PAB3D to 0.4 body lengths and coupled a full potential method to propagate to 3.6 body lengths.

\section{IV.E. Ames Low Boom Wing Tail (LBWT) with 4 Nacelles}

The Low Boom Wing Body Tail (LBWT) with nacelles and boundary layer diverters was chosen as a representative of a complete low sonic boom configuration, see Fig. 29 for the planform. The LBWT has four nacelles and a length of 12 inches. Cliff ${ }^{15}$ details the design of the configuration that was later utilized in a combined simulation and wind tunnel test campaign by Cliff, Baker, and Hicks. ${ }^{10}$ Further details of the wind tunnel test are provided by Mendoza, Hicks, and Cliff. ${ }^{59}$ Siclari and Fouladi60 also analyzed the LBWT with MIM3DSB.

The signatures from all methods are compared with experimental measurements digitized from Fig. 9(a) of Cliff, Baker, and Hicks. ${ }^{10}$ The Mach number is 2.0 and the angle of attack is 2.0 degrees. The lift coefficient varied between 0.070 and 0.080 in the wind tunnel test due to translating the configuration through test section flow angularity. The 2.0 degree angle of attack condition is set to simulate the average lift coefficient of 0.075 . The signature was measured at 14 inches below the nose, which is 1.167 body lengths. After the workshop, Durston et al. ${ }^{55}$ retested this configuration and measured similar signatures.

The measured signature is compared with the workshop methods in Fig. 30. As with the delta wing, this 


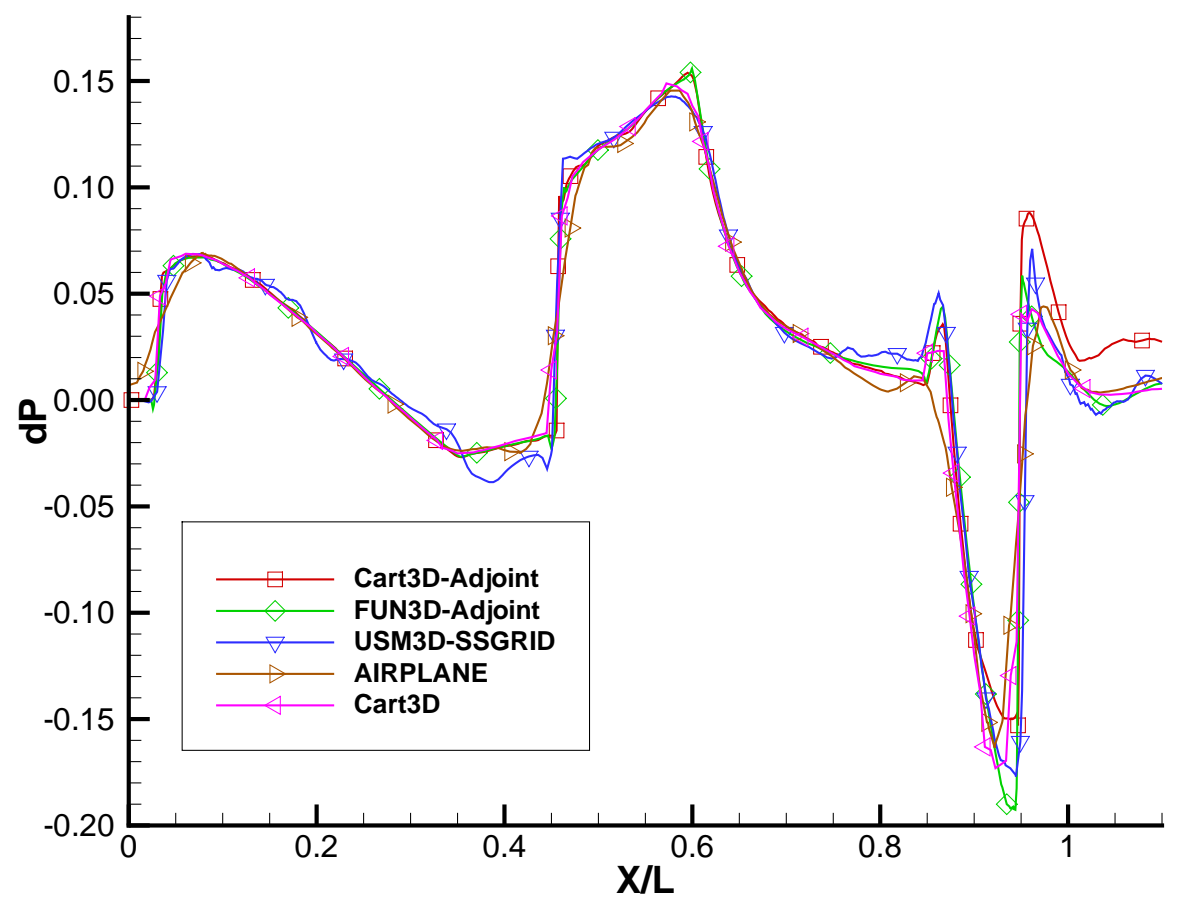

Figure 22. Nondimensional pressure signature for the delta wing body at $h / l=0.2$.

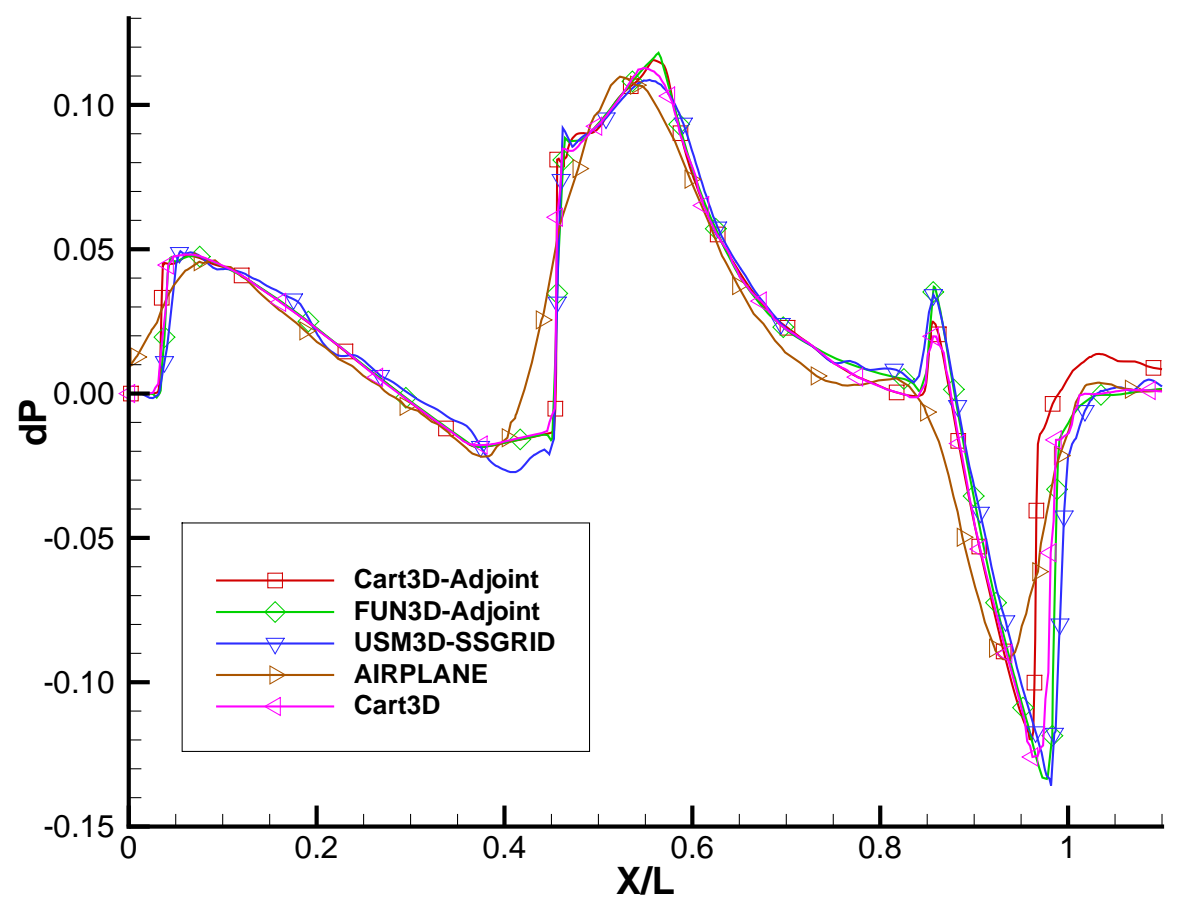

Figure 23. Nondimensional pressure signature for the delta wing body at $h / l=0.4$. 


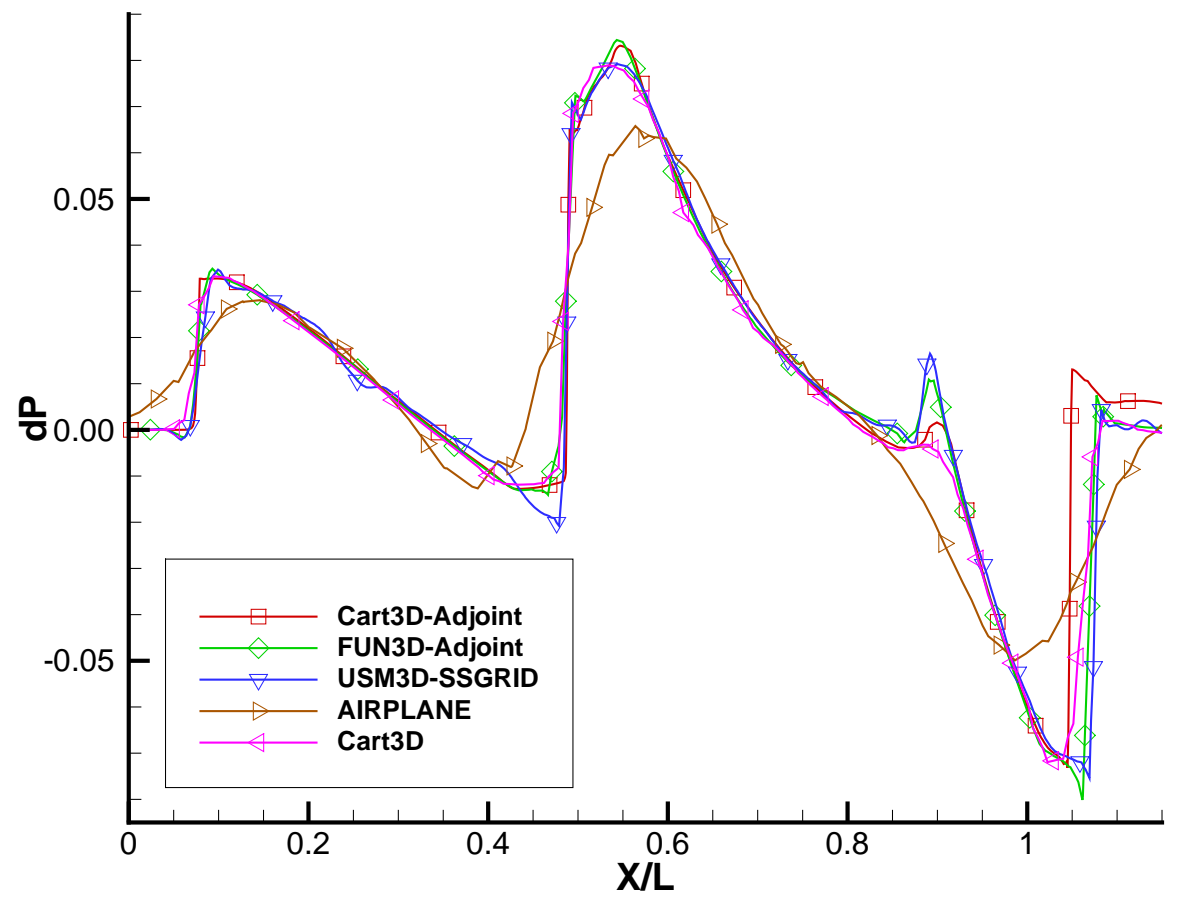

Figure 24. Nondimensional pressure signature for the delta wing body at $h / l=0.8$.

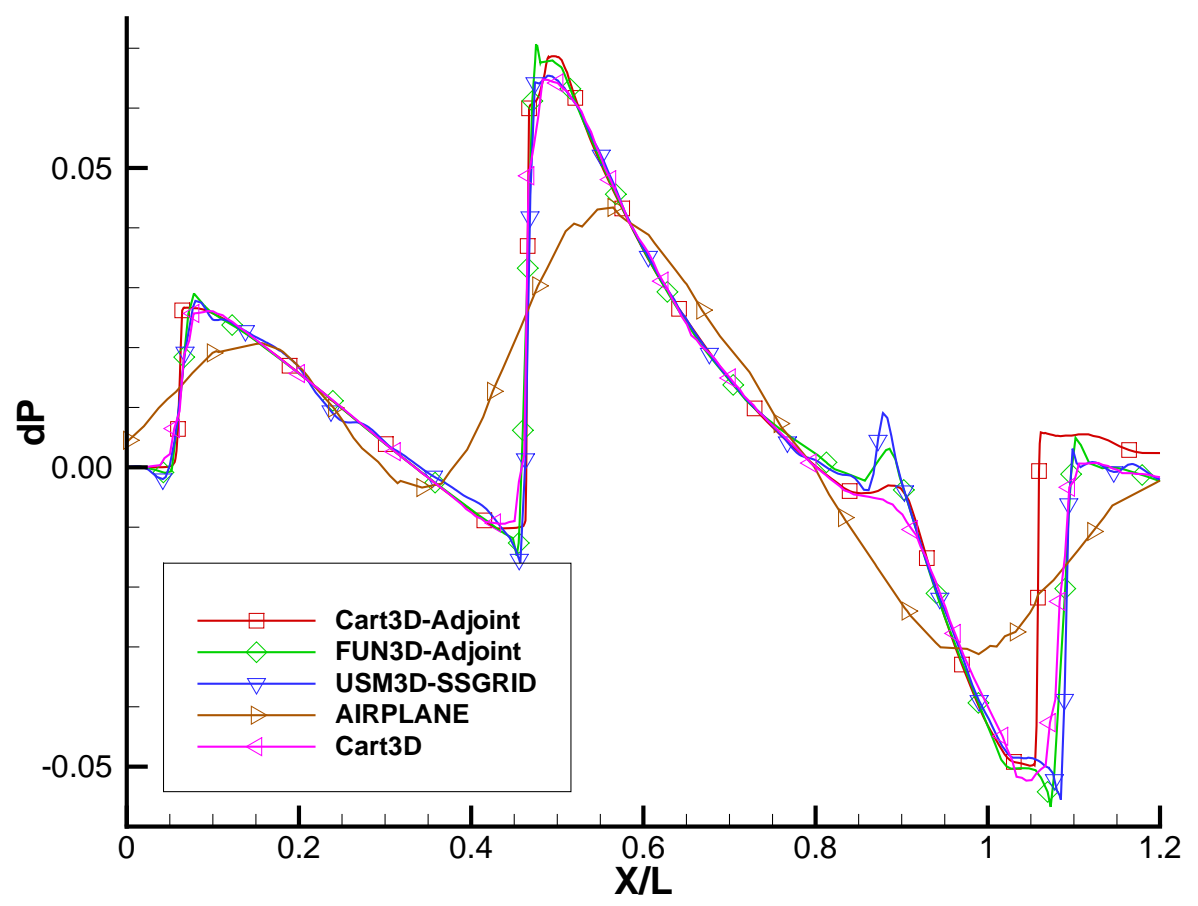

Figure 25. Nondimensional pressure signature for the delta wing body at $h / l=1.2$. 


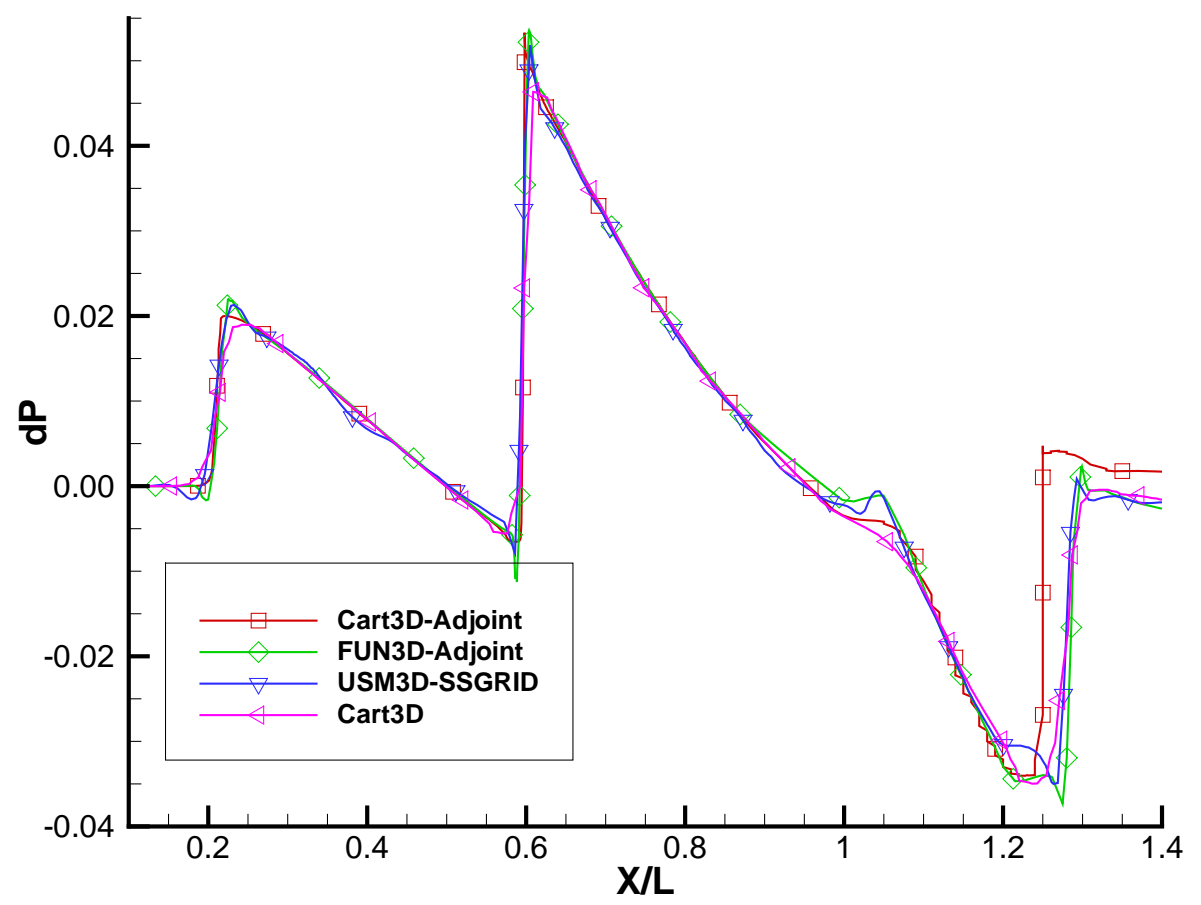

Figure 26. Nondimensional pressure signature for the delta wing body at $h / l=2.0$.

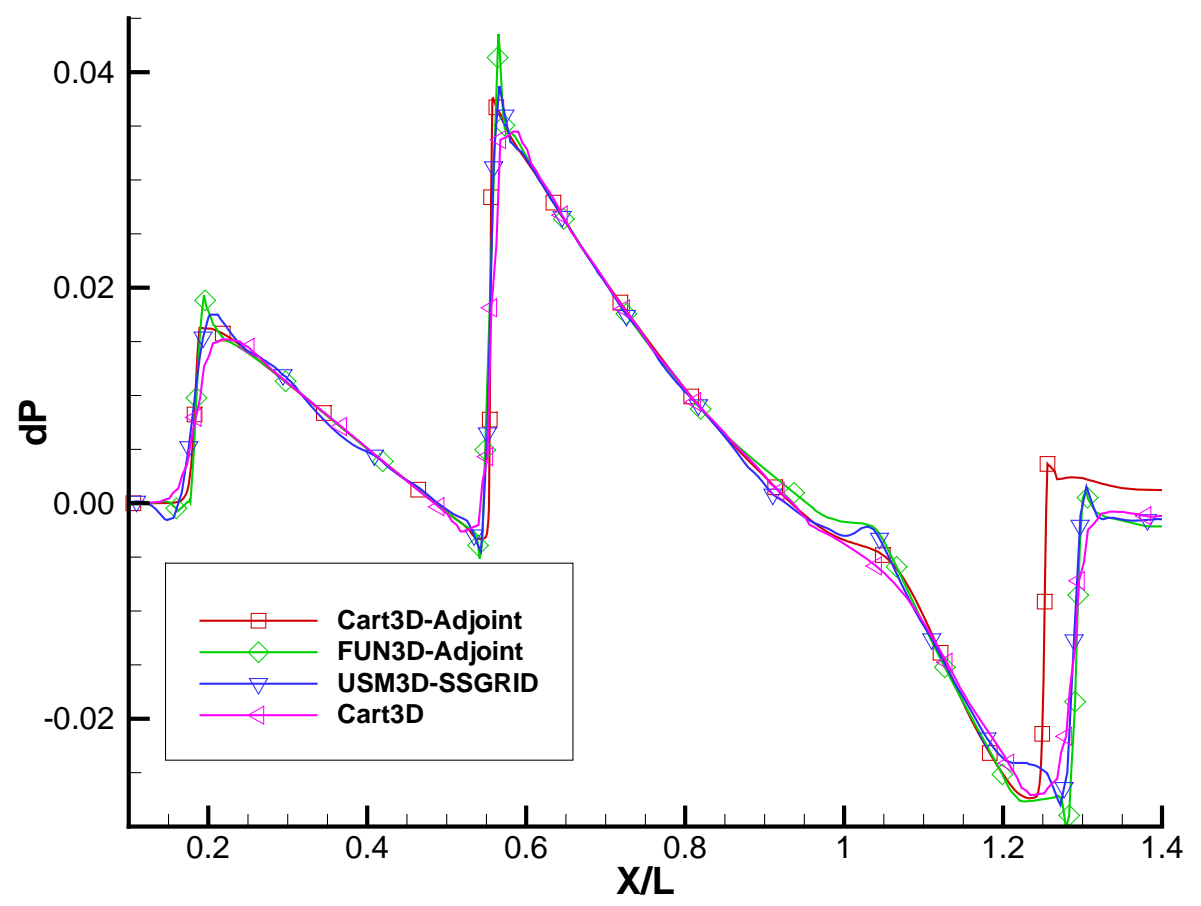

Figure 27. Nondimensional pressure signature for the delta wing body at $h / l=2.8$. 


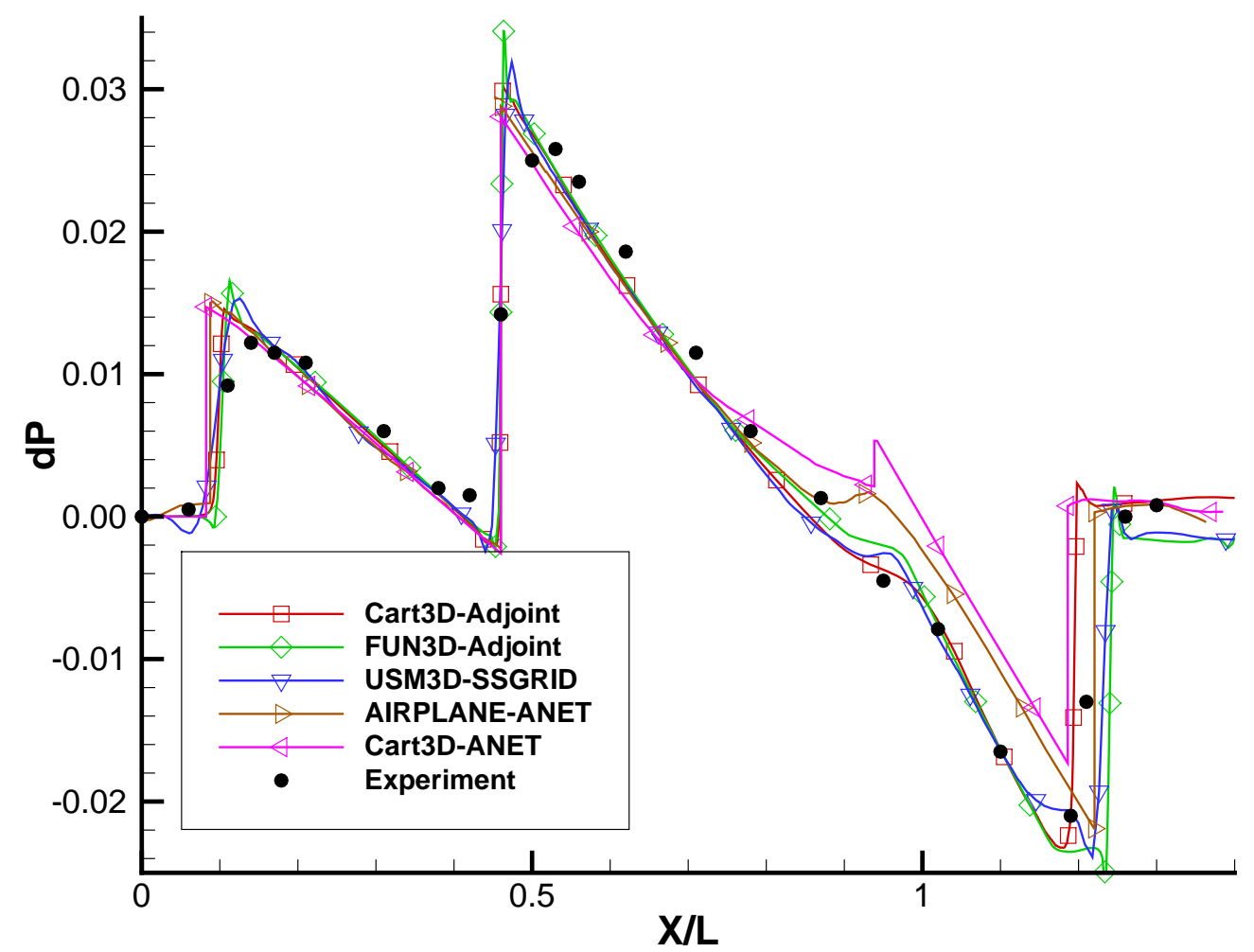

Figure 28. Nondimensional pressure signature for the delta wing body at $h / l=3.6$.

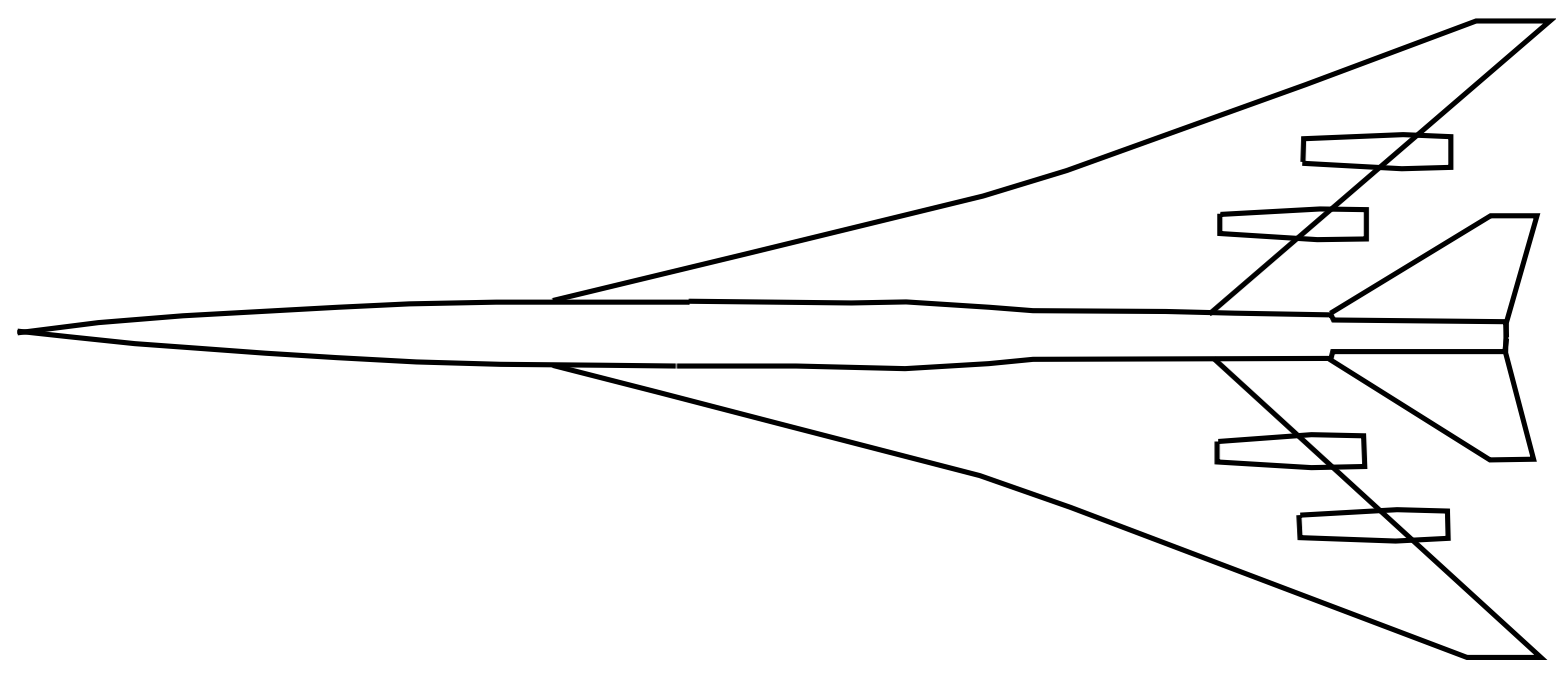

Figure 29. Planform of the LBWT fuselage, wings, tails, and engines.

\section{2 of 29}


signature lacks a simple expansion, so the signatures have been shifted slightly in $x / l$ to match pressure at the second compression attributed to the highly swept inboard wing, $x / l=0.75$. Details of the signatures aft of $x / l=1.0$ are provided using an expanded scale in Fig. 31. The various methods agree well with each other and the wind tunnel measurements forward of $x / l=1.1$. The location of the shock at $x / l=1.1$ is similar for all methods, but details aft of that location have a large degree of variation. These aft locations include the influence of the nacelles and boundary-layer diverters. As in the delta wing case, the various methods used slightly different geometries in the fuselage-sting juncture region. FUN3D-Adjoint predicted the most forward location of the shock at $x / l=1.2$. All of the workshop methods overpredicted the magnitude of this shock. Cart3D-Adjoint, FUN3D-Adjoint, and USM3D-SSGRID predict a small shock near $x / l=1.32$ This small shock was not predicted by the ANET methods. The wind tunnel measurements are too coarse to determine if this shock is present in the wind tunnel test. The location of the final shock near $x / l=1.45$ showed the largest variation.

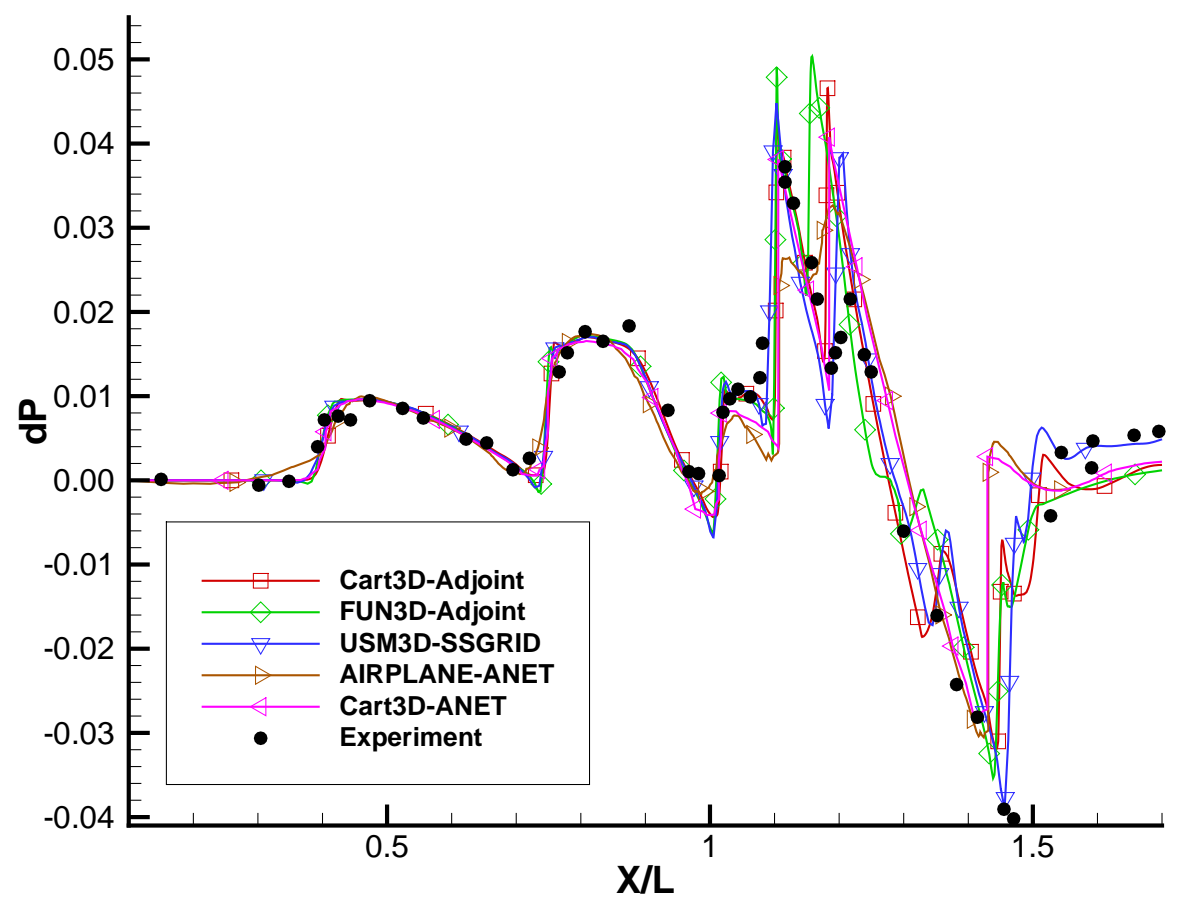

Figure 30. The entire nondimensional pressure signature for the LBWT at $h / l=1.167$.

\section{Execution Time}

The execution time required for each of the methods was gathered and reported at the workshop to gauge the resources required. Comparing the required resources is complicated because the analysis was performed on different computer architectures, see Table 3, with a wide range of grid sizes. The adjoint methods utilized adaptive schemes that included multiple flow and adjoint solves on increasing grid sizes. Estimates of the person-hours required to set up and run each of the cases was not recorded. Geometry set up and post processing time was also excluded.

Timing data for the cone cylinder, Table 4, and LBWT, Table 5, configurations is provided. Timing data was also presented at the workshop for the remaining cases, but is not provided here in the interest of brevity. These cases are representative of the resources required for the remaining cases. These are execution times as measured in 2008, on computer systems that are significantly slower than currently available systems. The grid generation time in minutes, the solution time in minutes, the solution time in number of $\mathrm{CPU}$ minutes, and the final number of control volumes in the grid is provided for each method. Cart3D-Adjoint 


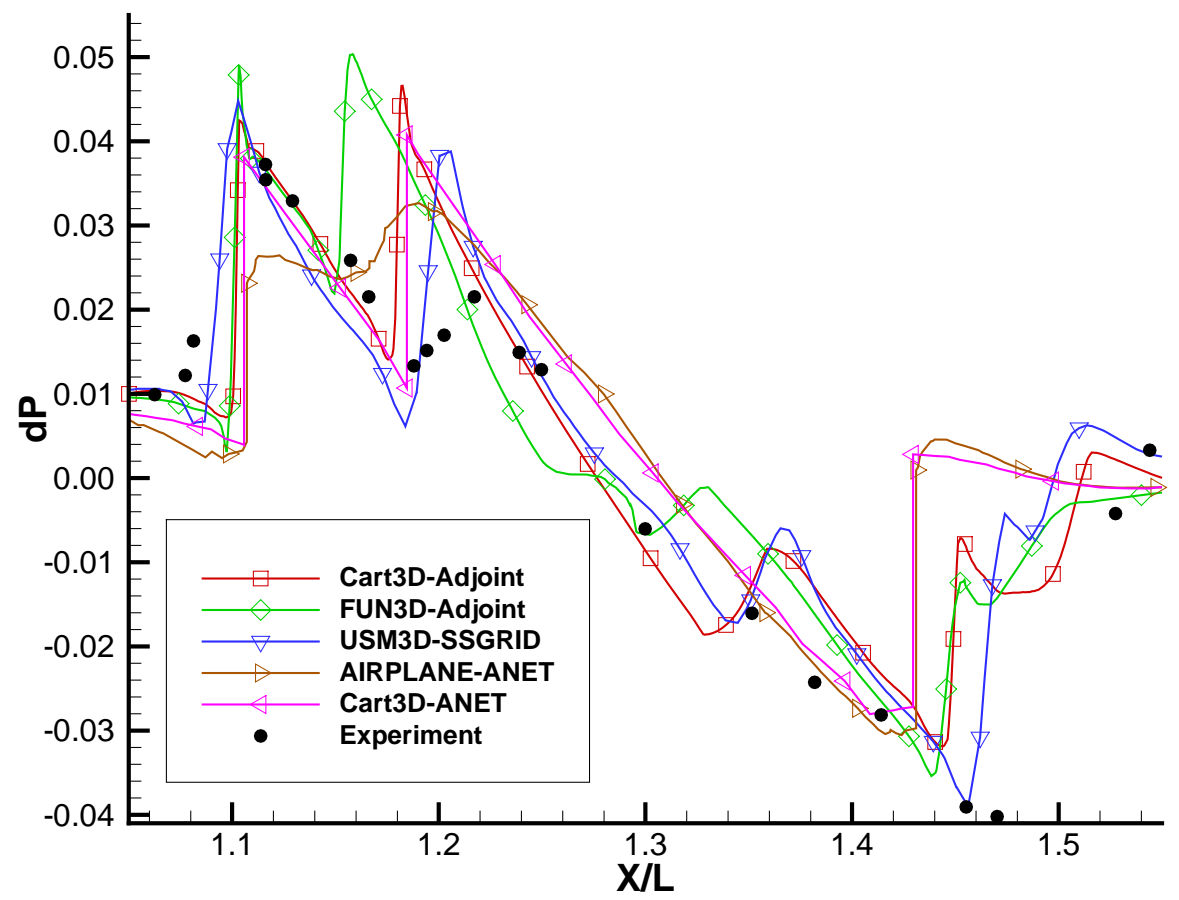

Figure 31. The center section of the nondimensional pressure signature for the LBWT at $h / l=1.167$.

Table 3. Computer architecture descriptions for workshop methods.

\begin{tabular}{lll} 
Method & Grid Generation & Solution \\
\hline Cart3D-Adjoint & 8 core Intel Xeon & 8 core Intel Xeon \\
& $3.2 \mathrm{Ghz}, 16 \mathrm{~GB}$ of memory & $3.2 \mathrm{Ghz}, 16 \mathrm{~GB}$ of memory \\
FUN3D-Adjoint & 1 Intel Pentium 4 & 24 Intel Pentium 4 \\
& $3.6 \mathrm{Ghz}, 2 \mathrm{~GB}$ of memory & $3.6 \mathrm{Ghz}, 2 \mathrm{~GB}$ of memory \\
AIRPLANE-ANET & 1 processor of a SGI Altix & 64 processors of a SGI Altix \\
& $1.5 \mathrm{Ghz}, 1 \mathrm{~TB}$ shared memory & $1.5 \mathrm{Ghz}, 1 \mathrm{~TB}$ shared memory \\
Cart3D-ANET & 1 processor of a SGI Altix & 64 processors of a SGI Altix \\
& $1.5 \mathrm{Ghz}, 1 \mathrm{~TB}$ shared memory & $1.5 \mathrm{Ghz}, 1 \mathrm{~TB}$ shared memory \\
USM3D-SSGRID & 1 core Intel Xeon & 48 processors of a SGI Altix \\
& $3 \mathrm{Ghz}, 16 \mathrm{~GB}$ of memory & $1.5 \mathrm{Ghz}, 1 \mathrm{~TB}$ shared memory
\end{tabular}


resources are the total of 3-5 flow solutions, adjoint solutions, and grid adaptation steps to reach the final grid. FUN3D-Adjoint resources are the total of 8-15 flow solutions, adjoint solutions, and grid adaptation steps to reach the final grid. Both the Cart3D-Adjoint and FUN3D-Adjoint methods have the option of adapting until a specified error level or grid size is reached. The other methods employ a manual grid size request to the grid generation software. Therefore, the exchange of accuracy for cost can be directly controlled by the user for any of these methods. Because of all the factors that affect the resources required for these methods, the information provided should only be used as a guideline and should be treated as extremely case specific and subject to the choices made by the analyst.

Table 4. Cone-cylinder execution time and grid size for workshop methods.

\begin{tabular}{lrrrr} 
Method & Grid Generation $(\mathrm{m})$ & Solution $(\mathrm{m})$ & Solution $(\mathrm{CPU} \times \mathrm{m})$ & Grid $(\mathrm{M})$ \\
\hline Cart3D-Adjoint & 2.9 & 38.1 & 305 & 3.29 \\
FUN3D-Adjoint & 1.0 & 240.0 & 5760 & 3.64 \\
AIRPLANE-ANET & 4.6 & 2.1 & 135 & 0.31 \\
Cart3D-ANET & 2.9 & 50.7 & 3246 & 10.24 \\
USM3D-SSGRID & 23.0 & 40.0 & 1920 & 9.70
\end{tabular}

Table 5. LBWT execution time and grid size for workshop methods.

\begin{tabular}{lrrrr} 
Method & Grid Generation $(\mathrm{m})$ & Solution $(\mathrm{m})$ & Solution $(\mathrm{CPU} \times \mathrm{m})$ & Grid $(\mathrm{M})$ \\
\hline Cart3D-Adjoint & 7.2 & 82.8 & 662 & 7.20 \\
FUN3D-Adjoint & 30.0 & 720.0 & 17280 & 6.94 \\
AIRPLANE-ANET & 12.7 & 3.7 & 234 & 1.06 \\
Cart3D-ANET & 3.1 & 48.4 & 3098 & 3.85 \\
USM3D-SSGRID & 50.0 & 127.0 & 6096 & 15.90
\end{tabular}

Cart3D-Adjoint required the least resources of the adaptive methods. Due to the differences in computer hardware, Cart3D-ANET required more CPU-minute resources for a single flow solution than all the flow and adjoint solutions of Cart3D-Adjoint with a final adapted grid of twice the size, see Table 5. The FUN3DAdjoint method required the most resources of the methods presented. An experimental cut-cell algorithm was used in this study, which did not have all of the optimzations that are available to the existing bodyfitted grid algorithm. AIRPLANE-ANET was the fastest method, but also utilized the smallest grids. It is possible that a finer grid may improve the AIRPLANE-ANET signatures presented at the workshop at the cost of a longer execution time. USM3D-SSGRID was near the middle of the required resources for some of the largest grids used in the workshop. An evaluation of the effects of reducing USM3D-SSGRID grid size indicated that the required resources can be reduced by as much as a factor of 5 with little loss of accuracy in the boom signatures. The differences in the signatures predicted by the current and reduced size grids is less than the differences between methods.

\section{Workshop Summary and Recommendations}

Five significantly different configurations were selected for the workshop to provide an accurate assessment of each of the workshop methods over a wide range of vehicles, Table 2. These configurations provided signatures with finite rise time, weak shocks, strong stocks, and their combination. Simple non-lifting to complex lifting configurations were chosen in an attempt to illustrate the differences in the methods. This allowed systematical and incremental increase in complexity toward relevant configurations.

A number of conclusions were drawn at the workshop. The most important is that any of the methods evaluated can produce reasonable results in hours making them suitable for full configuration analysis and design efforts. It was difficult to form succinct conclusions of the resources required by the methods because different grid sizes and computer architecture were employed. The accuracy of the available methods had improved and exceeded the expectations of the participants. The participants also reported that the automation of the methods has improved. The two adjoint-based adaptation techniques produced identical 
signatures, except in the extrema where limiter behavior is important or where the boundary conditions or geometry differed. This indicates that these two independent adaptive methods have reached an engineering level of grid convergence for 3D Euler flows.

The biggest concern of the workshop results was the scatter in the aft portion of the LBWT. The differences between the codes may indicate that the interactions of the fuselage, wing, tail, and nacelles is harder to predict than those items in isolation. To reduce wind tunnel measurement uncertainty, a recommendation was made to retest the LBWT to obtain higher fidelity wind tunnel data with more resolution of details of the multiple shock signature. Predicting the aft portion of the signatures for complex configuration was known to be a challenge before the workshop. ${ }^{4,18}$ Differences in the built and modeled geometry details or an unknown boundary layer state have been suspected as sources of uncertainty.

\section{Progress After Workshop}

Work has continued on improving sonic boom prediction and using these predictive tools in a design setting. Wayman et al. ${ }^{57}$ performed wind tunnel measurements that included the effect of boundary layer tripping. Significant changes in the aft portions of the signature were noted, which resulted in a recommendation to consider boundary layer state in future tests of wind tunnel models with transitional Reynolds numbers. Viscous effects have been included by multiple researchers. Carter, Campbell, and Nayani61 examined a low boom configuration with laminar and turbulent analysis. Elmiligui et al. ${ }^{62}$ applied Euler, laminar, and turbulent analysis that included the wind tunnel walls. Park ${ }^{63}$ has extended the 3D output-based adaptation technique to include viscous effects and examined this configuration. These computational efforts also demonstrated a sensitivity of the signature to analysis using Euler, laminar, and Reynolds-averaged Navier-Stokes with different turbulence models.

Grid generation schemes with a priori freestream shock alignment have been further improved. The MCAP collar grid approach was developed by Cliff et al. ${ }^{64}$ and applied the to delta wing and LBWT configurations. Elmiligui et al. ${ }^{65}$ applied MCAP to a wing body configuration. A new grid generation technique named Boom Grid (BG) also employs a prismatic extrusion approach. ${ }^{66}$

To better understand the propulsion effects on sonic boom, Bui, ${ }^{67}$ Castner, ${ }^{68}$ and Park and Carlson ${ }^{69}$ have included nozzle plumes in simulation. These propulsion effects have been incorporated into a design environment. Li et al. ${ }^{52}$ describes how SSGRID is used for sonic boom prediction including plume effects in an automated multidisciplinary design environment.

The adjoint-based grid adaptation schemes described at the workshop also enable gradient-based design. Aftosmis, Nemec, and Cliff ${ }^{5}$ utilized an adjoint-based design method to adjust aircraft outer mold line design to drive near-field signatures to a specified target. Rallabhandi, Nielsen, and Diskin ${ }^{70,71}$ have implemented a formal coupling of an adjoint Berger's boom propagation code to an adjoint CFD method. This allows aircraft outer mold line design to to drive ground signatures to a specified target or directly reduce A-weighted loudness on the ground.

\section{Conclusions}

A summary of the 2008 NASA Sonic Boom Prediction Workshop was provided. The five methods used on the five models were described. These models ranged from simple axisymmetric bodies to full lifting configurations. Using models with a range of complexity illustrated the applicability of the methods and aided identification of areas requiring further development, i.e., the aft signature of complex lifting configurations. The resources required by the methods was reported, but it was difficult to make direct comparisons because different grid sizes and computer architectures were employed by the participants. The workshop was placed in context with a history of methods available to NASA before the workshop and improvements that have been made after the workshop. All workshop methods were found to be sufficient for prediction of near-body signatures and useful for design. The largest difference between the simulated results was in the aft portion of the most complex configuration with wing, fuselage, tail, and nacelles. The simpler configurations with various strength shocks both lifting and non-lifting showed much better agreement between the methods.

\section{References}

${ }^{1}$ Bedard, Jr., A. J., "The Measurement of Sonic Boom Waveforms and Propagation Characteristics: Techniques and 
Challenges," AIAA Paper 90-4004, 1990.

${ }^{2}$ Plotkin, K. J., "State of the Art of Sonic Boom Modeling," Journal of the Acoustical Society of America, Vol. 111, No. 1, Jan. 2002, pp. 530-536.

${ }^{3}$ Ozcer, I. A., Sonic Boom Prediction Using Euler/Full-Potential Methodology, Master's thesis, Old Dominion University, Dec. 2005.

${ }^{4}$ Park, M. A., Anisotropic Output-Based Adaptation with Tetrahedral Cut Cells for Compressible Flows, Ph.D. thesis, Massachusetts Institute of Technology, Sept. 2008.

${ }^{5}$ Aftosmis, M. J., Nemec, M., and Cliff, S. E., "Adjoint-Based Low-Boom Design with Cart3D," AIAA Paper 2011-3500, 2011.

${ }^{6}$ Rallabhandi, S. K., "Advanced Sonic Boom Prediction Using the Augmented Burgers Equation," AIAA Journal of Aircraft, Vol. 48, No. 4, July-August 2011, pp. 1245-1253.

${ }^{7}$ Mendoza, J. P. and Hicks, R. M., "Further Studies of the Extrapolation of Near Field Overpressure Data," NASA TM-X-2219, NASA Ames Research Center, March 1971.

${ }^{8}$ Carlson, H., Mack, R., and Morris, O., "A Wind-Tunnel Investigation of the Effect of Body Shape on Sonic-Boom Pressure Distributions," NASA TN D-3106, NASA Langley Research Center, 1965.

${ }^{9}$ Hunton, L. W., Hicks, R. M., and Mendoza, J. P., "Some Effects of Wing Planform on Sonic Boom," NASA TN D-7160, NASA Ames Research Center, Jan. 1973.

${ }^{10}$ Cliff, S. E., Baker, T. J., and Hicks, R. M., "Design and Computational/Experimental Analysis of Low Sonic Boom Configurations," High-Speed Research: 1994 Sonic Boom Workshop: Configuration Design, Analysis, and Testing, edited by D. A. McCurdy, NASA CP-1999-209699, Dec. 1999, pp. 33-58.

${ }^{11}$ Thomas, C. L., "Extrapolation of Sonic Boom Pressure Signatures by the Waveform Parameter Method," NASA TN D-6832, June 1972.

${ }^{12}$ Cheung, S. H., Edwards, T. A., and Lawrence, S. L., "Application of Computational Fluid Dynamics to Sonic Boom Near- and Mid-Field Prediction," AIAA Journal of Aircraft, Vol. 29, No. 5, September-October 1992, pp. 920-926.

${ }^{13}$ Cliff, S. E. and Thomas, S. D., "Euler/Experiment Correlations of Sonic Boom Pressure Signatures," AIAA Journal of Aircraft, Vol. 30, No. 5, September-October 1993, pp. 669-675.

${ }^{14}$ Madson, M. D., "Sonic Boom Predictions Using a Solution-Adaptive Full-Potential Code," AIAA Journal of Aircraft, Vol. 31, No. 1, January-February 1994, pp. 57-63.

${ }^{15}$ Cliff, S. E., "On the Design and Analysis of Low Sonic Boom Configurations," High-Speed Research: Sonic Boom, Volume II, edited by T. A. Edwards, NASA CP-10133, Feb. 1994, pp. 37-78.

${ }^{16}$ Djomehri, M. J. and Erickson, L. L., "An Assessment of the Adaptive Unstructured Tetrahedral Grid, Euler Flow Solver Code FELISA," NASA TP-3526, NASA Ames Research Center, Dec. 1994.

${ }^{17}$ Fouladi, K., "Unstructured Grids for Sonic-Boom Analysis," AIAA Paper 99-2929, 1993.

${ }^{18}$ Carter, M. and Deere, K., "Grid Sourcing and Adaptation Study Using Unstructured Grids for Supersonic Boom Prediction," AIAA Paper 2008-6595, 2008.

${ }^{19}$ Campbell, R., Carter, M., Deere, K., and Waithe, K. A., "Efficient Unstructured Grid Adaptation Methods for Sonic Boom Prediction," AIAA Paper 2008-7327, 2008.

${ }^{20}$ Pirzadeh, S. Z., "Advanced Unstructured Grid Generation for Complex Aerodynamic Applications," AIAA Paper 20087178,2008

${ }^{21}$ Park, M. A., "Adjoint-Based, Three-Dimensional Error Prediction and Grid Adaptation," AIAA Journal, Vol. 42, No. 9, Sept. 2004, pp. 1854-1862.

${ }^{22}$ Lee-Rausch, E. M., Park, M. A., Jones, W. T., Hammond, D. P., and Nielsen, E. J., "Application of a Parallel AdjointBased Error Estimation and Anisotropic Grid Adaptation for Three-Dimensional Aerospace Configurations," AIAA Paper 2005-4842, 2005.

${ }^{23}$ Jones, W. T., Nielsen, E. J., and Park, M. A., "Validation of 3D Adjoint Based Error Estimation and Mesh Adaptation for Sonic Boom Prediction," AIAA Paper 2006-1150, 2006.

${ }^{24}$ Nemec, M., Aftosmis, M. J., and Wintzer, M., "Adjoint-Based Adaptive Mesh Refinement for Complex Geometries," AIAA Paper 2008-725, 2008.

${ }^{25}$ Aftosmis, M. J. and Berger, M. J., "Multilevel Error Estimation and Adaptive h-Refinement for Cartesian Meshes with Embedded Boundaries," AIAA Paper 2002-863, 2002.

${ }^{26}$ Nemec, M. and Aftosmis, M. J., "Adjoint Algorithm for CAD-Based Shape Optimization Using a Cartesian Method," AIAA Paper 2005-4987, 2005.

${ }^{27}$ Wintzer, M., Nemec, M., and Aftosmis, M. J., "Adjoint-Based Adaptive Mesh Refinement for Sonic Boom Prediction," AIAA Paper 2008-6593, 2008.

${ }^{28}$ Thomas, C. L., "Extrapolation of Wind Tunnel Sonic Boom Signatures Without use of a Waitham F-function," Third Conference on Sonic Boom Research, edited by I. R. Schwartz, NASA SP-225, Jan. 1971, pp. 205-217.

${ }^{29}$ Aftosmis, M. J., Berger, M. J., and Melton, J. E., "Robust and Effcient Cartesian Mesh Generation for Component-Based Geometry," AIAA Journal, Vol. 36, No. 6, 1998, pp. 952-960.

${ }^{30}$ van Leer, B., "Flux-Vector Splitting for the Euler Equations," Lecture Notes in Physics, Vol. 170, 1982, pp. $507-512$.

${ }^{31}$ Aftosmis, M. J., Berger, M. J., and Adomavicius, G., "A Parallel Multilevel Method for Adaptively Refined Cartesian Grids with Embedded Boundaries," AIAA Paper 2000-808, 2000.

${ }^{32}$ Aftosmis, M. J., Berger, M. J., and Murman, S. M., "Applications of Space-Filling Curves to Cartesian Methods for CFD," AIAA Paper 2004-1232, 2004.

${ }^{33}$ Berger, M. J., Aftosmis, M. J., and Murman, S. M., "Analysis of Slope Limiters on Irregular Grids," AIAA Paper 2005-490, 2005. 
${ }^{34}$ Cliff, S. E., Thomas, S. D., McMullen, M. S., Melton, J. E., and Durston, D. A., "Assessment of Unstructured Euler Methods for Sonic Boom Pressure Signatures Using Grid Refinement and Domain Rotation Methods," NASA TM-2008-214568, Sept. 2008.

${ }^{35}$ Jameson, A. and Baker, T. J., "Improvements to the Aircraft Euler Method," AIAA Paper 87-451, 1987.

${ }^{36}$ Baker, T. J., "Automatic Mesh Generation for Complex Three-Dimensional Regions Using a Constrained Delaunay Triangulation," Engineering with Computers, Vol. 5, 1989, pp. 161-175.

${ }^{37}$ Nemec, M. and Aftosmis, M. J., "Adjoint Error Estimation and Adaptive Refinement for Embedded-Boundary Cartesian Meshes," AIAA Paper 2007-4187, 2007.

${ }^{38}$ Venditti, D. A. and Darmofal, D. L., "Grid Adaptation for Functional Outputs: Application to Two-Dimensional Inviscid Flows," Journal of Computational Physics, Vol. 176, No. 1, 2002, pp. 40-69.

${ }^{39}$ Becker, R. and Rannacher, R., "An Optimal Control Approach to a posteriori Error Estimation in Finite Element Methods," Acta Numerica, Vol. 10, 2001, pp. 1-102.

${ }^{40}$ Giles, M. B. and Pierce, N. A., "Adjoint Error Correction for Integral Outputs," Error Estimation and Adaptive Discretization Methods in Computational Fluid Dynamics, edited by T. J. Barth and H. Deconinck, Springer, Dec. 2002, pp. $47-96$.

${ }^{41}$ Barth, T. J., "A Posteriori Error Estimation and Mesh Adaptivity for Finite Volume and Finite Element Methods," Adaptive Mesh Refinement - Theory and Applications, edited by T. Plewa, T. Linde, and V. G. Weirs, Vol. 41 of Lecture Notes in Computational Science and Engineering, Springer, 2005, pp. 183-202, Proceedings of the Chicago Workshop on Adaptive Mesh Refinement Methods, Sept. 3-5, 2003.

${ }^{42}$ Park, M. A. and Darmofal, D. L., "Validation of an Output-Adaptive, Tetrahedral Cut-Cell Method for Sonic Boom Prediction," AIAA Journal, Vol. 48, No. 9, Sept. 2010, pp. 1928-1945.

${ }^{43}$ Park, M. A. and Darmofal, D. L., "Parallel Anisotropic Tetrahedral Adaptation," AIAA Paper 2008-917, 2008.

${ }^{44}$ Venditti, D. A. and Darmofal, D. L., "Anisotropic Grid Adaptation for Functional Outputs: Application to TwoDimensional Viscous Flows," Journal of Computational Physics, Vol. 187, 2003, pp. 22-46.

${ }^{45}$ Park, M. A. and Darmofal, D. L., "Output-Adaptive Tetrahedral Cut-Cell Validation for Sonic Boom Prediction," AIAA Paper 2008-6594, 2008.

${ }^{46}$ Frink, N. T., Pirzadeh, S. Z., Parikh, P., Pandya, M. J., and Bhat, M. K., "The NASA Tetrahedral Unstructured Software System," The Aeronautical Journal, Vol. 104, No. 1040, Oct. 2000, pp. 491-499.

${ }^{47}$ Pirzadeh, S. Z., "Three-Dimensional Unstructured Viscous Grids by the Advancing-Layers Method," AIAA Journal, Vol. 34, No. 1, Jan. 1996, pp. 43-49.

${ }^{48}$ Frink, N. T., "Assessment of an Unstructured-Grid Method for Predicting 3-D Turbulent Viscous Flows," AIAA Paper 96-292, 1996.

${ }^{49}$ Roe, P. L., "Approximate Riemann Solvers, Parameter Vectors, and Difference Schemes," Journal of Computational Physics, Vol. 43, 1981, pp. 357-372.

${ }^{50}$ Samareh, J. A., "GridTool: A Surface Modeling and Grid Generation Tool," Proceedings of the Workshop on Surface Modeling, Grid Generation, and Related Issues in Computational Fluid Dynamic (CFD) Solutions, edited by Y. K. Choo, NASA CP-3291, March 1995, pp. 821-831.

${ }^{51}$ Pirzadeh, S. Z., "Structured Background Grids for Generation of Unstructured Grids by Advancing-Front Method," AIAA Journal, Vol. 31, No. 2, Feb. 1993, pp. 257-265.

${ }^{52} \mathrm{Li}$, W., Campbell, R., Geiselhart, K., Shields, E., Nayani, S., and Shenoy, R., "Integration of Engine, Plume, and CFD Analyses in Conceptual Design of Low-Boom Supersonic Aircraft," AIAA Paper 2009-1171, 2009.

${ }^{53}$ Morgenstern, J. M., "How to Accurately Measure Low Sonic Boom or Model Surface Pressures in Supersonic Wind Tunnels," AIAA Paper 2012-3215, 2012.

${ }^{54}$ Morgenstern, J. M., "Distortion Correction for Low Sonic Boom Measurement in Wind Tunnels," AIAA Paper 2012-3216, 2012.

${ }^{55}$ Durston, D. A., Cliff, S. E., Wayman, T. R., Merret, J. M., Elmiligui, A. A., and Bangert, L. S., "Near-Field Sonic Boom Test on Two Low-Boom Configurations Using Multiple Measurement Techniques at NASA Ames," AIAA Paper 2011-3333, 2011.

${ }^{56}$ Carlson, H. W., "Correlation of Sonic-Boom Theory with Wind-Tunnel and Flight Measurements," NASA TR-R-123, Dec. 1964.

${ }^{57}$ Wayman, T. R., Waithe, K. A., Howe, D. C., Bangert, L. S., and Wilcox, F., "Near Field Acoustic Test on a Low Boom Configuration in Langley's 4x4 Wind Tunnel," AIAA Paper 2011-3331, 2011.

${ }^{58}$ Kandil, O., Ozcer, I., Zheng, X., and Bobbitt, P., "Comparison of Full-Potential Propagation-Code Computations with the F-5E 'Shaped Sonic Boom Experiment' Program," AIAA Paper 2005-13, 2005.

${ }^{59}$ Mendoza, J. P., Hicks, R. M., and Cliff, S. E., "Wind Tunnel Sonic Boom Tests on Four High-Speed Civil Transport Models at Mach Numbers from 1.68 to 2.4," NASA TM-2008-214561, Sept. 2008.

${ }^{60}$ Siclari, M. J. and Fouladi, K., "A CFD Study of Component Configuration Effects on the Sonic Boom of Several HighSpeed Civil Transport Concepts," High-Speed Research: Sonic Boom, Volume II, edited by T. A. Edwards, NASA CP-10133, Feb. 1994, pp. 227-299.

${ }^{61}$ Carter, M. B., Campbell, R. L., and Nayani, S. N., "USM3D Analysis of Low Boom Configuration," AIAA Paper 2011-3335, 2011.

${ }^{62}$ Elmiligui, A., Cliff, S. E., Wilcox, F., Nemec, M., Bangert, L. S., Aftosmis, M. J., and Parlette, E., "Sonic Boom Computations for a Mach 1.6 Cruise Low Boom Configuraion and Comparisons with Wind Tunnel Data," AIAA Paper 20113496, 2011.

${ }^{63}$ Park, M. A., "Low Boom Configuration Analysis with FUN3D Adjoint Simulation Framework," AIAA Paper 2011-3337, 2011. 
${ }^{64}$ Cliff, S. E., Elmiligui, A. A., Campbell, R. L., and Thomas, S. D., "Evaluation of Refined Tetrahedral Meshes with Projected, Stretched, and Sheared Prism Layers for Sonic Boom Analysis," AIAA Paper 2011-3338, 2011.

${ }^{65}$ Elmiligui, A. A., Wilcox, F. J., Cliff, S., and Thomas, S., "Numerical Predictions of Sonic Boom Signatures for a Straight Line Segmented Leading Edge Model," Seventh International Conference on Computational Fluid Dynamics (ICCFD7), July 2012.

${ }^{66}$ Nayani, S. N. and Campbell, R. L., "New and Improved Grid Modification Methods for Low Boom Analysis," 51st Aerospace Sciences Meeting, Grapevine, Texas, American Institute of Aeronautics and Astronautics, Reston, VA (submitted for publication).

${ }^{67}$ Bui, T. T., "Computational Fluid Dynamics Analysis of Nozzle Plume Effects on Sonic Boom Signature," AIAA Journal of Aircraft, Vol. 48, No. 2, March-April 2011, pp. 368-380.

${ }^{68}$ Castner, R. S., "Exhaust Nozzle Plume Effects on Sonic Boom," AIAA Journal of Aircraft, Vol. 49, No. 2, March-April 2012, pp. 415-422.

${ }^{69}$ Park, M. A. and Carlson, J.-R., "Turbulent Output-Based Anisotropic Adaptation," AIAA Paper 2010-168, 2010.

${ }^{70}$ Rallabhandi, S. K., "Sonic Boom Adjoint Methodology and its Applications," AIAA Paper 2011-3497, 2011.

${ }^{71}$ Rallabhandi, S. K., Nielsen, E. J., and Diskin, B., "Sonic Boom Mitigation Through Aircraft Design and Adjoint Methodology," AIAA Paper 2012-3220, 2012. 